\title{
Toric K3-fibred Calabi-Yau manifolds with del Pezzo divisors for string compactifications
}

\author{
Michele Cicoli, ${ }^{a}$ Maximilian Kreuzer and Christoph Mayrhofer ${ }^{b}$ \\ ${ }^{a}$ Deutsches Elektronen-Synchrotron DESY, \\ Notkestrasse 85, 22607 Hamburg, Germany \\ ${ }^{b}$ Institut für Theoretische Physik, Universität Heidelberg, \\ Heidelberg, Germany \\ E-mail: michele.cicoli@desy.de, c.mayrhofer@thphys.uni-heidelberg.de
}

ABstract: We analyse several explicit toric examples of compact K3-fibred Calabi-Yau three-folds. These manifolds can be used for the study of string dualities and are crucial ingredients for the construction of LARGE Volume type IIB vacua with promising applications to cosmology and particle phenomenology. In order to build a phenomenologically viable model, on top of the two moduli corresponding to the base and the K3 fibre, we demand also the existence of two additional rigid divisors: the first supporting the nonperturbative effects needed to achieve moduli stabilisation, and the second allowing the presence of chiral matter on wrapped D-branes. We clarify the topology of these rigid divisors by discussing the interplay between a diagonal structure of the Calabi-Yau volume and D-terms. Del Pezzo divisors appearing in the volume form in a completely diagonal way are natural candidates for supporting non-perturbative effects and for quiver constructions, while 'non-diagonal' del Pezzo and rigid but not del Pezzo divisors are particularly interesting for model building in the geometric regime. Searching through the existing list of four dimensional reflexive lattice polytopes, we find 158 examples admitting a Calabi-Yau hypersurface with a K3 fibration and four Kähler moduli where at least one of the toric divisors is a 'diagonal' del Pezzo. We work out explicitly the topological details of a few examples showing how, in the case of simplicial polytopes, all the del Pezzo divisors are 'diagonal', while 'non-diagonal' ones appear only in the case of non-simplicial polytopes. A companion paper will use these results in the study of moduli stabilisation for globally consistent explicit Calabi-Yau compactifications with the local presence of chirality.

Keywords: Flux compactifications, Differential and Algebraic Geometry, Superstring Vacua 


\section{Contents}

1 Introduction $\quad 2$

2 Diagonal divisors $\quad 5$

2.1 Kähler moduli of Calabi-Yau compactifications 6

2.2 Diagonalisation of the volume form 6

$3 \quad \boldsymbol{D}$-terms and shrinking cycles $\quad 8$

3.1 Fluxed $D$ branes and Fayet-Iliopoulos terms 9

$\begin{array}{ll}3.2 & \text { Shrinking divisors }\end{array}$

$\begin{array}{lll}3.3 & D \text {-terms and geometric regime } & 11\end{array}$

4 The search for suitable K3 fibrations 12

$5 \quad$ K3 fibrations from simplicial polytopes 13

5.1 The exemplar: $\mathbb{P}_{[1,1,2,2,2]}^{4}(8) / \mathbb{Z}_{2}: 01100 \mathrm{~T} 1$

5.1.1 Divisor analysis 16

$\begin{array}{lll}5.1 .2 & \text { Orientifolding } & 18\end{array}$

$\begin{array}{lll}6 & \text { K3 fibrations from non-simplicial polytopes } & 18\end{array}$

$\begin{array}{lll}\text { 6.1 A K3 fibration with two del Pezzo divisors } & 19\end{array}$

$\begin{array}{lll}6.1 .1 & \text { Divisor analysis } & 21\end{array}$

6.1.2 Orientifolding 22

6.2 A K3 fibration with one del Pezzo divisor 23

6.2.1 Divisor analysis 25

$\begin{array}{ll}6.2 .2 & \text { Orientifolding }\end{array}$

$\begin{array}{llr}7 & \text { Conclusions } & 27\end{array}$

A Two K3 fibrations with an F-theory uplift 28

A.1 $\mathbb{P}_{[1,1,1,1,4]}^{4}(8)$ with three blow-ups $\quad 28$

$\begin{array}{ll}\text { A.1.1 Divisor analysis } & 30\end{array}$

$\begin{array}{ll}\text { A.1.2 Orientifolding } & 30\end{array}$

A.2 Another example with one del Pezzo divisor 30

$\begin{array}{ll}\text { A.2.1 Divisor analysis } & 32\end{array}$

$\begin{array}{lll}\text { A.2.2 Orientifolding } & 33\end{array}$

B List of all the K3 fibrations with diagonal del Pezzo divisors 33 


\section{Introduction}

K3 fibrations play a crucial rôle in the study of string compactifications. Historically they have been first used to study mirror symmetry for two-parameter Calabi-Yau manifolds built as hypersurfaces embedded in complex weighted projective spaces [1].

In [2] it has then been realised that K3 fibrations are a fundamental ingredient to understand strong-weak coupling dualities between $4 \mathrm{D} \mathcal{N}=2$ supersymmetric theories which are the low energy result of string compactifications [3,4]. Subsequently, it has been shown that if some strongly coupled $\mathcal{N}=2$ heterotic vacua admit a dual description in terms of weakly coupled type IIA string theory compactified on a Calabi-Yau three-fold, the compactification manifold has to be a K3 fibration [5] (see also [6-9] for other studies of string dualities based on K3 fibrations).

The original work of [2] provided a short list of K3-fibred Calabi-Yau three-folds, consisting of 31 hypersurfaces embedded in weighted $\mathbb{P}^{4}$ and 25 complete intersections in weighted $\mathbb{P}^{5}$. These results were generalised in [10] where the authors found 628 examples identifying all the K3 fibrations which can be built as transverse polynomials in weighted $\mathbb{P}^{4}$. The powerful methods of toric geometry allowed the authors of [11] to provide many more explicit constructions of K3-fibred Calabi-Yau three-folds. In fact, they found 124,701 examples of $\mathrm{K} 3$ fibrations realised as hypersurfaces embedded in toric varieties.

More recently, K3-fibred Calabi-Yau three-folds received new attention in the context of type IIB orientifold compactifications. Here all the closed string moduli, but those parameterising the Kähler deformations of the Calabi-Yau metric, can be stabilised by turning on background fluxes [12].

Very interesting vacua among this kind of string constructions are the so-called LARGE Volume Scenarios (LVS), which are characterised by the presence of a non-supersymmetric minimum at exponentially large volume of the compactification manifold $[13,14]$. The simplest LVS, originally proposed in [13], is based on type IIB compactified on an orientifold of the Calabi-Yau $\mathbb{P}_{[1,1,1,6,9]}^{4}(18)$. This Calabi-Yau admits only two Kähler moduli which can be stabilised by the interplay of non-perturbative and $\alpha^{\prime}$ corrections without the need to fine-tune the background fluxes. The internal volume $\mathcal{V}=\tau_{b}^{3 / 2}-\tau_{s}^{3 / 2}$ is controlled by one exponentially large 4-cycle $\tau_{b} \sim e^{c / g_{s}} \gg 1$ (for $c \sim \mathcal{O}(1)$ and $g_{s}<1$ in order to trust perturbation theory). The second Kähler modulus $\tau_{s} \sim 1 / g_{s}>1$ controls the volume of a small blow-up mode. The fact that the Calabi-Yau volume is exponentially large guarantees that the approximations in the effective field theory are under good control and allows to generate several interesting phenomenological hierarchies.

This simple construction can be generalised adding more blow-up modes which can all be fixed at relatively small values by non-perturbative effects [15]. The Calabi-Yau acquires a distinctive 'Swiss-cheese' shape where the overall volume, $\mathcal{V}=\tau_{b}^{3 / 2}-\sum_{i} \tau_{s, i}^{3 / 2}$, is still controlled by just one four-cycle $\tau_{b}$ which can be arbitrarily large while the various small four-cycles $\tau_{i}$ control the size of the 'holes' of the Swiss-cheese. Examples with $h^{1,1}=3$ having this form are the Fano three-fold $\mathcal{F}_{11}$ [16], the degree 15 hypersurface embedded in $\mathbb{P}_{[1,3,3,3,5]}^{4}$ and the degree 30 hypersurface in $\mathbb{P}_{[1,1,3,10,15]}^{4}[17]$ while several examples with $h^{1,1}=4$ have been provided in [18]. 
The first examples of LVS with a topological structure more complicated than the Swiss-cheese one, were discovered in [14]. The authors assumed the existence of a K3fibred Calabi-Yau with $h^{1,1}=3$, obtained by adding an ad hoc blow-up mode to the geometry $\mathbb{P}_{[1,1,2,2,6]}^{4}(12)$ studied in [1]. Now the Calabi-Yau volume is not controlled anymore by just one large four-cycle, but by two four-cycles: the K3 fibre, $\tau_{1}$, and another four-cycle, $\tau_{2}$, which contains the $\mathbb{P}^{1}$ base $t_{1}$ of the fibration. Hence it takes the form $\mathcal{V}=\sqrt{\tau_{1}} \tau_{2}-\tau_{s}^{3 / 2}=t_{1} \tau_{1}-\tau_{s}^{3 / 2}$ with $\tau_{2}=t_{1} \sqrt{\tau_{1}}$ (the generalisation to models with many blow-up modes is straightforward).

The fact that the internal volume is controlled by two four-cycles turns out to be very promising for both cosmological and phenomenological applications for the following reasons:

- Given that $\alpha^{\prime}$ and non-perturbative effects fix the overall volume exponentially large, only the combination $\sqrt{\tau_{1}} \tau_{2} \sim e^{c / g_{s}} \gg 1$ gets stabilised leaving a flat direction (the small blow-up mode is still fixed at relatively small values $\tau_{s} \sim 1 / g_{s}>1$ ). Other stabilising effects come from string loop corrections to the Kähler potential but it has been shown that for the original $\mathbb{P}_{[1,1,1,6,9]}^{4}(18)$ model they turn out to be subleading with respect to the other corrections [19]. The authors of [20] then showed that $g_{s}$ corrections turn out generically to be subdominant with respect to $\alpha^{\prime}$ and nonperturbative effects. This is due to a leading order cancellation of the $g_{s}$ contribution to the scalar potential, called 'extended no-scale structure'.

Therefore, in the K3-fibred case, there is a direction in the $\left(\tau_{1}, \tau_{2}\right)$-plane orthogonal to the overall volume which is naturally flatter than all the other ones. This remaining flat direction can be lifted only when subleading string loops are taken into account. In fact, these corrections give rise to a minimum located at $\tau_{1} \sim g_{s}^{4 / 3} \mathcal{V}^{2 / 3}[14]$.

Due to the shallowness of this direction, this scalar field is a natural candidate for cosmological applications [21, 22]. In [23] it has been used as an inflaton field, in a large-field inflationary model which predicts observably large gravitational waves and where the $\eta$-problem can be solved without requiring fine-tuning. Subsequently, this direction has also been exploited as a curvaton field, leading to an interesting model which predicts detectable non-Gaussianities of local type [24].

Other interesting cosmological applications of LARGE Volume K3-fibred compactifications involve the study of reheating [25, 26] and finite-temperature effects [27].

- The base $\times$ fibre structure of the volume gives the possibility to take the anisotropic limit $t_{1} \gg \sqrt{\tau_{1}}$ (or $\tau_{2} \gg \tau_{1}$ ), corresponding to interesting geometries having the two dimensions of the base, spanned by the cycle $t_{1}$, larger than the other four of the K3 fibre, spanned by $\tau_{1}$.

These anisotropic compactifications turn out to be very promising to make contact with current experiments from the study of the phenomenological properties of hidden Abelian gauge bosons with a kinetic mixing with the ordinary photon. In fact, they lead naturally to dark forces for an intermediate string scale, or even to a hidden 
CMB allowing some fine-tuning of the underlying parameters for the extreme case of TeV-scale strings [28].

Moreover, it has been recently shown that the moduli can indeed be stabilised in this very anisotropic region of the Kähler cone [29]. This leads to the first stringy derivation of popular models with effectively two large extra dimensions of micron-size and fundamental gravity scale around a few $\mathrm{TeV}$ [30-33]. The use of K3-fibred compactifications is needed not only to obtain an anisotropic shape of the Calabi-Yau but also to generate the poly-instantons effects [34] which are crucial to fix the K3 divisor at relatively small values $\tau_{1} \sim 1 / g_{s}>1$ like a blow-up mode of the previous examples.

Despite all these phenomenological successes, these models still lack a rigorous mathematical foundation, in the sense that no explicit Calabi-Yau examples of K3 fibrations with additional blow-up modes are known in the literature yet. The goal of this paper is exactly to fill in this gap by using the powerful methods of toric geometry.

We shall search for K3-fibred Calabi-Yau three-folds which satisfy the two topological conditions for the existence of a LVS minimum derived in [14]:

1. The Euler number of the Calabi Yau manifold must be negative, or more precisely: $h^{1,2}>h^{1,1}>1$

2. The Calabi-Yau manifold must have at least one 'diagonal' blow-up mode.

We shall discuss the second condition and understand its geometrical meaning more in depth. In fact, in section 2 we shall notice that there can be three types of 'blow-up' divisors:

- 'Diagonal' del Pezzo: these four-cycles are truly local effects, in the sense that it can always be found a basis where this divisor $D_{\mathrm{dP}}$ has only its triple self intersection number non-vanishing, or equivalently where $\sqrt{\operatorname{Vol}\left(D_{\mathrm{dP}}\right)} \propto \operatorname{Vol}\left(D_{\mathrm{dP}} \cap D_{\mathrm{dP}}\right)$. This conditions implies that this del Pezzo four-cycle will appear in the volume form in a completely diagonal manner. It can also be shown to correspond to the condition on the inverse Kähler metric $K_{\mathrm{dP}, \mathrm{dP}}^{-1}=\mathcal{V} \sqrt{\tau_{\mathrm{dP}}}$, where $\tau_{\mathrm{dP}}=\operatorname{Vol}\left(D_{\mathrm{dP}}\right)$. This condition has been used in [14] to infer that the divisor $D_{\mathrm{dP}}$ has to come from a resolution of a point-like singularity. However this requirement is too restrictive since we are aware of examples of del Pezzo divisors satisfying $\sqrt{\operatorname{Vol}\left(D_{\mathrm{dP}}\right)} \propto \operatorname{Vol}\left(D_{\mathrm{dP}} \cap D_{\mathrm{dP}}\right)$ but which give rise to a smooth space when they are shrunk to zero size. The reason is that not just singularities can be blown-up, but also any smooth point.

Besides being the natural candidate for supporting the non-perturbative effects of the LVS, this kind of divisor is very useful also for chiral model building. In fact, in section 3 we shall show that the constraint coming from the vanishing $D$-term condition, in the absence of singlets which can acquire non-vanishing vacuum expectation values, always forces this four-cycles to shrink to zero size. This naturally leads to quiver GUT-like constructions [35, 36].

- 'Non-diagonal' del Pezzo: these four-cycles are characterised by the fact that it is impossible to find a basis where they do not intersect any other divisor. Given that 
they are not genuinely local effects, they will not appear in the Calabi-Yau volume in a completely diagonal way, corresponding to a form of the relative elements of the inverse Kähler metric which renders these four-cycles inappropriate for supporting the non-perturbative effects needed for the realisation of the LVS.

On the contrary, these divisors turn out to be the best candidates to support GUT constructions with magnetised branes wrapping cycles stabilised in the geometric regime (see [37] for the type IIB case and [38, 39] for the F-theory case). For rigid divisors no unwanted matter in the adjoint representation gets generated. Furthermore, in this 'non-diagonal' case, the vanishing $D$-term condition may not force the shrinking of any cycle. However, the final answer to this question is very modeldependent given that some examples of this kind are known where the $D$-terms still lead the system to the boundary of the Kähler cone [18].

- Rigid but not del Pezzo: there can finally be other rigid four-cycle with $h^{1,0}=h^{2,0}=0$ which are not del Pezzo divisors, meaning that they are not contractible to a point. An intuitive way to visualise these four-cycles, is to think about them as blow-ups of curves. Similarly to the 'non-diagonal' del Pezzo divisors, they also cannot be used to support the non-perturbative effects in the LVS, but are natural candidates for GUT model building in the geometric regime.

With this classification of blow-up cycles at hands, we performed a search through the existing list of four dimensional reflexive lattice polytopes [40, 41] for K3-fibred Calabi-Yau three-folds with $h^{1,1}=4$ where at least one of the toric divisors is a 'diagonal' del Pezzo divisor. As we have already pointed out, this condition is needed in order to obtain the LVS. On top of the presence of the two moduli corresponding to the base and the K3 fibre and the four-cycle for the non-perturbative effects, we also demanded the presence of a second rigid divisor for chiral model building.

The final result of our scan, summarised in section 4, consists of 158 examples of lattice polytopes. In the case of simplicial polytopes, all the del Pezzo four-cycles of each CalabiYau example are 'diagonal' divisors, as can be seen in the illustrative exemplar worked out in detail in section 5 .

In the $h^{1,1}=4$ case, 'non-diagonal' del Pezzo divisors appear only in the case of nonsimplicial polytopes. We discuss them in section 6 . There, we present two examples of K3 fibrations, the first with two del Pezzo four-cycles whereas the second with just a single del Pezzo divisor. Two more explicit examples are described in appendix A. Finally in appendix B we present the full list of all the 158 examples of lattice polytopes which have a Calabi-Yau hypersurface with $h^{1,1}=4$ that admits a K3 fibration structure and at least one 'diagonal' del Pezzo.

\section{Diagonal divisors}

In this section we shall present a procedure to single out the divisors which enter the volume form in a completely diagonal way [42], and so which are the natural candidates to support the non-perturbative effects of the LVS. 


\subsection{Kähler moduli of Calabi-Yau compactifications}

Before describing the details of the diagonalisation procedure, let us set our notations and conventions focusing on the case of type IIB flux compactifications on an orientifold of a Calabi-Yau three-fold $Y_{3}$, which preserves $\mathcal{N}=1$ supersymmetry in 4D [12, 43, 44].

Considering orientifold projections such that $h_{-}^{1,1}=0 \Rightarrow h_{+}^{1,1}=h^{1,1}$, the Kähler form, $J$, can be expanded in a basis $\left\{\hat{D}_{i}\right\}_{i=1}^{h^{1,1}}$ of $H^{1,1}\left(Y_{3}, \mathbb{Z}\right)$ as:

$$
J=t^{i} \hat{D}_{i} .
$$

Each two-form $\hat{D}_{i}$ is Poincaré dual to the divisor $D_{i}$ on $\mathcal{X}$, i.e.:

$$
\int_{\mathcal{X}} \omega \wedge \hat{D}_{i}:=\int_{D_{i}} \omega \quad \forall \omega \in H^{\operatorname{dim}(\mathcal{X})-2}(\mathcal{X}, \mathbb{Z}) \quad \text { and } \quad D_{i} \in H_{\operatorname{dim}(\mathcal{X})-2}(\mathcal{X}, \mathbb{Z}),
$$

where $\mathcal{X}$ can be a Calabi-Yau hypersurface $Y_{3}$ or its toric ambient space $X_{4}$. We shall keep the 'hat' in this section but drop it in the following, given that it should always be clear from the context whether it is a divisor or its Poincaré dual two-form.

The volume of the internal manifold takes the form:

$$
\mathcal{V}=\frac{1}{3 !} \int_{Y_{3}} J \wedge J \wedge J=\frac{1}{3 !} k_{i j k} t^{i} t^{j} t^{k}
$$

Here $k_{i j k}$ are the triple intersection numbers of $Y_{3}$ given by:

$$
k_{i j k}=\int_{Y_{3}} \hat{D}_{i} \wedge \hat{D}_{j} \wedge \hat{D}_{k}=D_{i} \cdot D_{j} \cdot D_{k}
$$

while the $t^{i} \in \mathbb{R}$ parameterise 2-cycle volumes. Note that the $t^{i}$ are not restricted to the positive real numbers since the $\hat{D}_{i}$ are not in general the generators of the Kähler cone.

The quantities $t^{i}$ are not the correct bosonic components of the chiral multiplets of the low-energy $\mathcal{N}=14 \mathrm{D}$ supergravity. In fact, the Kähler moduli are given by the $T_{i}=\tau_{i}+\mathrm{i} b_{i}$ which are related to the $t^{i}$ as follows: $\tau_{i}$ is the Einstein-frame volume (in units of $l_{s}$ ) of the divisor $D_{i} \in H_{4}\left(Y_{3}, \mathbb{Z}\right)$ and reads:

$$
\tau_{i}=\frac{1}{2} \int_{D_{i}} \imath^{*} J \wedge \imath^{*} J=\frac{1}{2} \int_{Y_{3}} \hat{D}_{i} \wedge J \wedge J=\frac{\partial \mathcal{V}}{\partial t^{i}}=\frac{1}{2} k_{i j k} t^{j} t^{k},
$$

where $\imath^{*} J$ is the pullback of $J$ to $D_{i}$. Its axionic partner $b_{i}$ is the component of the Ramond-Ramond 4-form $C_{4}$ along this cycle: $\int_{D_{i}} \imath^{*} C_{4}=b_{i}$.

The tree-level Kähler potential $K_{\text {tree }}=-2 \ln \mathcal{V}$ is given as a function of $T_{i}$ by solving the equations (2.5) for the $t^{i}$ as functions of the $\tau_{i}=\frac{1}{2}\left(T_{i}+\bar{T}_{i}\right)$, and substituting the result into eq. (2.3) to evaluate $\mathcal{V}$.

\subsection{Diagonalisation of the volume form}

We shall now look for a change of basis $\left\{\hat{D}_{i}\right\}_{i=1}^{h^{1,1}}$ to $\left\{\hat{D}_{i}^{\prime}\right\}_{i=1}^{h^{1,1}}$ which brings the volume, or part of it, in a diagonal form. Parameterising this change of basis in terms of an $\left(h^{1,1} \times h^{1,1}\right)$ matrix $a$ of generic integer coefficients $a_{i}{ }^{j}$, the new basis elements can be expressed as $\hat{D}_{i}^{\prime}=a_{i}{ }^{j} \hat{D}_{j}$ with corresponding volumes given by:

$$
\tau_{i}^{\prime}=\frac{1}{2} \int_{Y_{3}} \hat{D}_{i}^{\prime} \wedge J \wedge J=\frac{1}{2} a_{i}{ }^{j} k_{j p q} t^{p} t^{q} .
$$


We now search for vectors $\vec{a}_{i} \in \mathbb{Z}^{h^{1,1}}$ such that the divisor volumes $\tau_{i}^{\prime}$ become a complete square in terms of the $t^{i}$. If there exist any such $\vec{a}_{i}$, we call the number of linear independent solutions $n_{s} \leq h^{1,1}$. We take these solutions as the first $n_{s}$ rows of $a$, while the remaining $\vec{a}_{j}$, with $n_{s}<j \leq h^{1,1}\left(Y_{3}\right)$, give the completion of $a$ to a full rank matrix. In the case $n_{s}=h^{1,1}$ the volume form can be completely diagonalised, resulting in a 'strong' Swiss-cheese CalabiYau, while for $n_{s}<h^{1,1}$ we shall simply speak of 'weak' Swiss-cheese manifolds.

In order to find such solutions, we consider the symmetric matrices:

$$
\left(A_{i}\right)_{p q}:=\frac{1}{2} a_{i}{ }^{j} k_{j p q},
$$

which admit a similarity transformation to the diagonal matrices $A_{i}^{\prime}$ :

$$
A_{i}^{\prime}=\left(\begin{array}{ccccc}
\lambda_{i 1} & \cdots & \cdots & \cdots & 0 \\
\vdots & \ddots & & & \vdots \\
\vdots & & \lambda_{\text {in }} & & \vdots \\
\vdots & & & \ddots & \vdots \\
0 & \cdots & \cdots & \cdots & \lambda_{i h^{1,1}}
\end{array}\right)
$$

The divisor volume $\tau_{i}^{\prime}=\left(A_{i}\right)_{p q} t^{p} t^{q}$ becomes a complete square only if the matrix $A_{i}$ has just one non-zero eigenvalue $\lambda_{\text {in }}$ plus $\left(h^{1,1}-1\right)$ vanishing eigenvalues $\lambda_{\text {im }}$ for all $m \neq n$.

Given that the eigenvalues of $A_{i}$ are functions, or more precisely sections, of the $h^{1,1}$ integer vectors $\vec{a}_{i}$, we have to find a solution to:

$$
\lambda_{m}\left(\vec{a}_{i}\right)=\lambda_{i m}=0 \quad \forall m \neq n \quad \text { and } \quad \lambda_{n}\left(\vec{a}_{i}\right)=\lambda_{\text {in }} \neq 0 .
$$

If there exist any solution to (2.9), we can have two situations:

1. The inner product between the eigenvector $\vec{v}_{\text {in }}$, corresponding to the only nonvanishing eigenvalue $\lambda_{\text {in }} \neq 0$, and $\vec{a}_{i}$ does not vanish:

$$
\left\langle\vec{a}_{i}, \vec{v}_{\mathrm{in}}\right\rangle:=a_{i}{ }^{j}\left(\vec{v}_{\mathrm{in}}\right)_{j} \neq 0 .
$$

In this case we can expand $\vec{a}_{i}$ as a sum over $\vec{v}_{\text {in }}$ and its normal space $\left\{\vec{v}_{i m}\right\}_{m \neq n}^{h^{1,1}}$ corresponding to the zero eigenvalues:

$$
\vec{a}_{i}=\frac{\left\langle\vec{a}_{i}, \vec{v}_{\text {in }}\right\rangle}{\left\langle\vec{v}_{\text {in }}, \vec{v}_{\text {in }}\right\rangle} \vec{v}_{\text {in }}+\sum_{r \neq n}^{h^{1,1}} c_{r} \vec{v}_{i r} .
$$

Therefore we can rewrite the intersection polynomial for every solution $i$ in the following form:

$$
I_{3}=\lambda_{\mathrm{in}} \frac{\left\langle\vec{a}_{i}, \vec{v}_{\mathrm{in}}\right\rangle^{2}}{\left\langle\vec{v}_{\mathrm{in}}, \vec{v}_{\mathrm{in}}\right\rangle} D_{i}^{\prime} D_{i}^{\prime} D_{i}^{\prime}+k_{r k l}^{\prime} D_{r}^{\prime} D_{k}^{\prime} D_{l}^{\prime},
$$

with $D_{i}^{\prime}=a_{i}{ }^{j} D_{j}$ and $D_{r}^{\prime}=\left(\vec{v}_{i r}\right)^{j} D_{j}$, where $j$ runs from 1 to $h^{1,1}$, but $r, k$ and $l$ omit $n$. If $n_{s}>1$ we see from:

$$
\left(\vec{v}_{k n}\right)_{p} \propto\left(A_{k}\right)_{p q} a_{i}{ }^{q}=\frac{1}{2} a_{k}{ }^{j} k_{j p q} a_{i}^{q}=\left(A_{i}\right)_{j p} a_{k}{ }^{j} \propto\left(\vec{v}_{\mathrm{in}}\right)_{p},
$$


that $\vec{a}_{i}$ has to be normal to all $\vec{v}_{k n}$ with $k \neq i$. Hence we obtain a basis in which the intersection polynomial further simplifies to:

$$
I_{3}=\sum_{i=1}^{n_{s}} \lambda_{i n} \frac{\left\langle\vec{a}_{i}, \vec{v}_{i n}\right\rangle^{2}}{\left\langle\vec{v}_{i n}, \vec{v}_{i n}\right\rangle} D_{i}^{\prime} D_{i}^{\prime} D_{i}^{\prime}+\sum_{p, q, r=1}^{h^{1,1}-n_{s}} k_{p q r}^{\prime} D_{p}^{\prime} D_{q}^{\prime} D_{r}^{\prime},
$$

where now each $D_{p}^{\prime}, p=1, \ldots,\left(h^{1,1}-n_{s}\right)$, is generated by a basis element of the space normal to the linear span of the $\vec{v}_{i n}$ with $i=1, \ldots, n_{s}$. We wrote out the sums explicitly to indicate that we sum also over $i$, in contrast to above.

2. In the case when $\left\langle\vec{a}_{i}, \vec{v}_{i n}\right\rangle=0, \vec{a}_{i}$ belongs to the normal space to $\vec{v}_{i n}$. Hence the intersection polynomial takes the form:

$$
I_{3}=\lambda_{\text {in }}\left\langle\vec{v}_{\text {in }}, \vec{v}_{\text {in }}\right\rangle^{2} D_{i}^{\prime} D_{n}^{\prime} D_{n}^{\prime}+\sum_{p, q, r \neq l, n}^{h^{1,1}} k_{p q r}^{\prime} D_{p}^{\prime} D_{q}^{\prime} D_{r}^{\prime},
$$

where $D_{i}^{\prime}=a_{i}{ }^{j} D_{j} \propto\left(\vec{v}_{i l}\right)^{j} D_{j}, D_{n}^{\prime}=\left(\vec{v}_{\text {in }}\right)^{j} D_{j}$, and each $D_{p}^{\prime}$, with $n \neq p \neq l$, is generated by basis elements of the space normal to the linear span of $\vec{a}_{i} \propto \vec{v}_{i l}$ and $\vec{v}_{\text {in. }}$. For $n_{s}>1$ we may obtain combinations of (2.14) and (2.15).

We notice that if the divisor $D_{i}^{\prime}$ is numerically effective then the linearity of the intersection form in $D_{i}^{\prime}$, together with the fact that $D_{i}^{\prime} \cdot c_{2}\left(Y_{3}\right)>0$, implies that the Calabi-Yau is a fibration over $\mathbb{P}^{1}$ with generic fibre given by a K3 surface [45].

From this discussion, we conclude that for a divisor $D$ with: ${ }^{1}$

$$
\tau_{D} \propto \operatorname{Vol}(D \cap D)^{2}=\left(\int_{D \cap D} J\right)^{2},
$$

we can find a basis of divisors such that $I_{3}=k_{D} D \cdot D \cdot D+\ldots$ where the ellipsis denote intersections independent of $D$. In this basis the constant of proportionality in (2.16) becomes:

$$
\operatorname{Vol}(D \cap D)^{2}=2 k_{D} \operatorname{Vol}(D) .
$$

This is the condition that defines what we called a 'diagonal' four-cycle. Moreover, if not obstructed by the Kähler cone, it implies the existence of a decoupling limit for $D$ where:

$$
\lim _{J \rightarrow J_{0}} \operatorname{Vol}(D)=0 \neq\left.\operatorname{Vol}(D)\right|_{J} \simeq \mathcal{O}(1), \quad \text { and }\left.\quad \lim _{J \rightarrow J_{0}} \operatorname{Vol}\left(Y_{3}\right) \simeq \operatorname{Vol}\left(Y_{3}\right)\right|_{J} \gg \mathcal{O}(1)
$$

\section{$3 \quad$ D-terms and shrinking cycles}

In this section we shall study the interplay between a diagonal structure of the CalabiYau volume and the requirement of having vanishing Fayet-Iliopoulos terms, showing that 'diagonal' del Pezzo divisors are always forced to shrink to zero size.

\footnotetext{
${ }^{1}$ Note that the curve $D \cap D$ may not be effective.
} 


\subsection{Fluxed $D$ branes and Fayet-Iliopoulos terms}

A very important ingredient of type IIB flux compactifications is the presence of spacetime filling $D 7$-branes which can wrap an internal divisor $D_{i}$. Each $D 7$-brane comes along with a $\mathrm{U}(1)$ gauge theory that lives on its eight-dimensional world-volume $\mathcal{W}$. The brane is embedded in the ten-dimensional space-time manifold $\mathcal{X}_{10}=\mathbb{R}^{3,1} \times Y_{3}$ via the map $\imath: \mathcal{W} \hookrightarrow \mathcal{X}_{10}$.

Furthermore, the $D 7$-brane wrapping the divisor $D_{i}$ can be fluxed, in the sense that we can turn on a generic internal magnetic flux $F_{i}$. The flux can be expanded as [46]:

$$
F_{i}=f_{i}^{k} \imath^{*} D_{k}+f_{i}^{a} \omega_{a}
$$

with $D_{k} \in H^{1,1}(X)$ and $\omega_{a} \in H^{1,1^{\perp}}\left(D_{i}\right)$ where $H^{1,1}\left(D_{i}\right)=\imath^{*} H^{1,1}(X) \oplus H^{1,1^{\perp}}\left(D_{i}\right)$ and $\imath^{*}$ denotes the pullback operation. $H^{1,1^{\perp}}\left(D_{i}\right)$ is the space of $(1,1)$-forms which are defined on the world-volume of the $D 7$-brane wrapping $D_{i}$ such that $\int_{D_{i}} \imath^{*} D_{j} \wedge \omega_{a}=0 \forall j$ and $a$.

This gauge flux on $D_{i}$ induces a U(1)-charge $q_{i j}$ for the Kähler modulus $T_{j}$ which takes the form:

$$
q_{i j}=\int_{D_{i}} \imath^{*} D_{j} \wedge\left(F_{i}-\imath^{*} B\right)=\tilde{f}_{i}^{k} \int_{Y_{3}} D_{i} \wedge D_{j} \wedge D_{k}=\tilde{f}_{i}^{k} k_{i j k},
$$

where we have absorbed the $B$-field coefficients in $\tilde{f}_{i}^{k}$. The Kähler modulus $T_{\mathrm{U}(1)_{i}}$ which gets coupled to the $\mathrm{U}(1)$ gauge boson living on $D_{i}$, is in general a combination of divisors corresponding to the four-cycle Poincaré dual to the two-cycle supporting the magnetic flux. Due to this coupling, the U(1) gauge boson becomes massive via the Stückelberg mechanism by eating the axion $a_{\mathrm{U}(1)_{i}}=\operatorname{Im}\left(T_{\mathrm{U}(1)_{i}}\right)$, but $\tau_{\mathrm{U}(1)_{i}}=\operatorname{Re}\left(T_{\mathrm{U}(1)_{i}}\right)$ remains as a light modulus in the effective field theory and gives rise to a moduli-dependent FayetIliopoulos (FI) term $\xi_{i}$ which looks like [47-49]:

$$
\xi_{i}=\frac{1}{4 \pi \mathcal{V}} \int_{D_{i}} \imath^{*} J \wedge\left(F_{i}-\imath^{*} B\right)=\frac{1}{4 \pi \mathcal{V}} t^{j} \tilde{f}_{i}^{k} k_{i j k}=\frac{q_{i j}}{4 \pi} \frac{t^{j}}{\mathcal{V}}=-\frac{q_{i j}}{4 \pi} \frac{\partial K}{\partial \tau_{j}}
$$

Therefore we end up with a $D$-term scalar potential of the form $V_{D}=\frac{g_{i}^{2}}{2} \xi_{i}^{2}$ where the gauge coupling constant $g_{i}$ is given by (considering the dilaton $\phi$ fixed at its VEV: $e^{\phi}=g_{s}$ ):

$$
\frac{2 \pi}{g_{i}^{2}}=\operatorname{Re}\left(T_{i}\right)-\frac{\tilde{f}_{i}^{j} q_{i j}}{2 g_{s}}
$$

Including also the presence of unnormalised charged matter fields $\varphi_{j}$ (open string states) with corresponding $\mathrm{U}(1)$ charges given by $c_{i j}$, the resulting $D$-term potential looks like:

$$
V_{D}=\frac{g_{i}^{2}}{2}\left(\sum_{j} c_{i j} \varphi_{j} \frac{\partial K}{\partial \varphi_{j}}-\xi_{i}\right)^{2}=\frac{2 \pi g_{s}}{2 \tau_{i} g_{s}-\tilde{f}_{i}^{j} q_{i j}}\left(\sum_{j} c_{i j} \varphi_{j} \frac{\partial K}{\partial \varphi_{j}}+\frac{q_{i j}}{4 \pi} \frac{\partial K}{\partial \tau_{j}}\right)^{2} .
$$

\subsection{Shrinking divisors}

The $D$-term potential is particularly important for the stabilisation of the four-cycle $\tau_{i}$ which supports a Standard Model-like brane construction with chiral matter given that 
this modulus cannot be fixed by non-perturbative effects [17]. ${ }^{2}$ In fact, the presence of chiral matter with vanishing VEVs generically kills the instanton contribution to the superpotential.

Other possible contributions to fix $\tau_{i}$ are string loop corrections. However, the $D$-terms are the leading effect in a large volume expansion since they scale as $\mathcal{V}^{-2}$ whereas the string loop corrections behave as $\mathcal{V}^{-3} \tau_{i}^{-1 / 2}$.

Moreover, the standard LVS potential generated by non-perturbative and $\alpha^{\prime}$ corrections, behaves like $\mathcal{V}^{-3}$. Hence the $D$-term potential has to cancel (at least at leading order up to $\mathcal{O}\left(\mathcal{V}^{-3}\right)$ ) in order not to develop a run-away direction for the volume mode. In the absence of Standard Model singlets which can acquire non-zero VEVs and for vanishing VEVs of visible sector fields, the supersymmetric requirement of having $V_{D}=0$ implies $\xi=0$. We shall now show that in the case of a 'diagonal' del Pezzo this requirement forces this cycle to shrink to zero size [37].

First of all we impose the requirement of having no chiral intersections between the visible $D 7$-stack wrapped around $D_{i}$ and the ED3 instanton wrapped around a generic rigid divisor $D_{E 3}$, which is needed to generate the non-perturbative effects of the LVS:

$$
\left\langle\Gamma_{i}, \Gamma_{E 3}\right\rangle=\int_{Y_{3}} D_{i} \wedge D_{E 3} \wedge\left(F_{i}-B\right)=\tilde{f}_{i}^{j} k_{i j E 3}=q_{i E 3}=0
$$

where the total world-volume flux on the instanton is zero: $\mathcal{F}_{E 3}=F_{E 3}-B=0 .{ }^{3}$ This requirement simplifies the expression (3.3) for the FI term to:

$$
\xi_{i}=\frac{1}{4 \pi \mathcal{V}} \sum_{j \neq E 3} q_{i j} t^{j}=-\frac{1}{4 \pi} \sum_{j \neq E 3} q_{i j} \frac{\partial K}{\partial \tau_{j}} .
$$

This expression further simplifies if $D_{i}$ is a 'diagonal' del Pezzo since its defining relation (2.17) implies that $k_{i j k} \neq 0$ only if $i=j=k$, or, in terms of $\mathrm{U}(1)$-charges, $q_{i j} \neq 0$ only if $i=j$. Hence (3.7) becomes:

$$
\xi_{i}=\frac{q_{i i} t^{i}}{4 \pi \mathcal{V}}=c_{i} \sqrt{\tau_{i}}
$$

where:

$$
c_{i}=\frac{\tilde{f}_{i}^{i}}{4 \pi \mathcal{V}} \sqrt{2 k_{i i i}} \neq 0 .
$$

Therefore if we do not want to lead the system to any decompactification limit corresponding to $\mathcal{V} \rightarrow \infty, \xi_{i}=0$ implies $\tau_{i}^{\prime} \rightarrow 0$. This result is in agreement with previous explicit Calabi-Yau examples. In fact, the authors of [37] presented an example with a diagonalisable volume which is suitable to realise the LVS but where all the rigid divisors are $\mathrm{dP}_{8}$ four-cycles which are forced to shrink to a point by the $D$-terms. Similar situations where found in [18] where the authors presented several Calabi-Yau examples with a 'strong'

\footnotetext{
${ }^{2}$ This might not be the case for compactifications with $h_{-}^{1,1} \neq 0[50]$.

${ }^{3}$ We recall that the cycle wrapped by the ED3 is a rigid non-Spin four-cycle, and so we always need to turn on a half integer flux in order to cancel the Freed-Witten anomalies: $F_{E 3}=(1 / 2) D_{E 3}$. This flux can then be compensated by the $B$-field.
} 
Swiss cheese structure. However, only some of the diagonal divisor were del Pezzo and could be contracted without interfering with the volume of the other divisors. All cases were promising to realise the LVS but there were always one or more four-cycles shrinking to zero size due to the supersymmetric condition $D=0$.

The shrinking of a cycle is generically considered as a problem because it is hard to have control over $\alpha^{\prime}$ and quantum corrections at the singular regime, while the effective field theory in the geometric regime is much more under control. However if one does not care that much about this control issue, then the shrinking of a cycle can even be welcomed in order to reproduce the nice phenomenological features of models at the quiver locus [35]. Some of these constructions with fractional branes at singularities allow the sequestering of the visible sector with a hierarchy between $M_{\text {soft }}$ and $m_{3 / 2}, M_{\text {soft }} \sim m_{3 / 2}^{2} / M_{P}$, which might lead naturally to both GUT-theories and TeV-scale SUSY [36].

Therefore we showed how a 'diagonal' del Pezzo divisor is the best candidate either to support the non-perturbative effects of the LVS (in this case no FI-term gets generated) or to lead the system to the quiver locus.

\section{3 $\quad D$-terms and geometric regime}

We have argued that every time the branes involved in the GUT construction wrap divisors appearing in the volume in a diagonal manner, then the vanishing FI term condition, always forces some cycles to shrink to zero size.

Let us now try to discuss possible ways to avoid this shrinking limit so that the visible sector can be built via magnetised branes wrapping cycles in the geometric regime:

1. Consider brane constructions with Standard Model singlets (like a right handed sneutrino) which can get a non-vanishing VEV cancelling the FI term: $\langle|\varphi|\rangle \sim \sqrt{\xi}$. In this way the FI term is not forced to zero anymore and the cycle supporting the visible sector could then be fixed using string loop corrections to the Kähler potential [14].

2. Wrap around the shrinking cycle the instanton giving rise to the top Yukawa coupling [37, 51]. More precisely, an $O(1)$ instanton wrapping a rigid cycle invariant under the orientifold action and with the following chiral intersections with the GUT stack $D 7_{a}$ and the intersecting stack $D 7_{b}$ :

$$
I_{a, \text { inst }}=1, \quad I_{b, \text { inst }}=-1,
$$

generates perturbatively forbidden top-Yukawa couplings:

$$
Y_{\alpha \beta} \mathbf{1 0} \mathbf{1 0}^{\beta} \mathbf{5}_{H} e^{-\tau_{\text {inst }},}
$$

which are exponentially suppressed, and so very far from their $\mathcal{O}(1)$ phenomenological values. This is one of the main reasons for considering F-theory instead of type IIB GUT constructions. However if the instanton cycle shrinks to zero size, then the top-Yukawa coupling becomes phenomenologically viable: $e^{-\tau_{\text {inst }}} \rightarrow 1$ for $\tau_{\text {inst }} \rightarrow 0$.

3. Consider Calabi-Yau three-folds without a diagonal structure for the cycles supporting the branes involved in the GUT construction, but still with a rigid divisor which 
is orthogonal to all these four-cycles and appears in the volume in a diagonal way. This further cycle is needed to get the LVS.

The authors of [37] presented a case where all rigid divisors are $\mathrm{dP}_{9}$, hence they are not contractible to a point. ${ }^{4}$ The volume does not have a diagonal structure, and so there is no shrinking cycle due to $D$-terms. However the LVS could not be realised due to the absence of a 'diagonal' del Pezzo.

4. Place the GUT theory on the K3 fibre which does not appear in the volume in a diagonal manner, and so the $D$-terms might not force any cycle to contract to a point. However the K3 fibre deformations should be lifted in order not to get unwanted matter in the adjoint representation. Moreover, contrary to previous studies [14], the K3 fibre should be stabilised at a relatively small value in order not to get an exponentially small value for the gauge coupling of the visible sector.

\section{The search for suitable K3 fibrations}

In this section we describe how to search for Calabi-Yau hypersurfaces that admit a K3 fibration and a 'weak' Swiss-cheese type volume structure. As we have already pointed out, this kind of manifolds are needed to realise particular versions of the LVS which are very interesting for their applications to cosmology [23-25, 27] and particle phenomenology $[28,29]$.

The first input parameter for our search is the number of Kähler moduli $h^{1,1}$ of the Calabi-Yau $Y_{3}$. Moduli stabilisation requires already the presence of a third modulus, a 'diagonal' del Pezzo, in addition to the two moduli controlling the volume of the base and the K3-fibre. We focus however on $h^{1,1}=4$ since we are interested in finding a second rigid divisor for a chiral $D$-brane configuration.

Furthermore, we want to use toric techniques to control all the Kähler moduli (see $[52,53]$ for a mathematical description of the notation and the necessary tools, or [54-58] for more physical introductions to this topic). Therefore, we take into account only models that have all their Picard generators induced from the toric ambient four-fold $X_{4}$. In the heterotic literature, these manifolds are called favourable [59].

With these input data, we perform our search without starting from the list of K3fibred toric Calabi-Yau hypersurfaces [11] but, for convenience and statistical reasons, we prefer to scan the list of four-dimensional reflexive lattice polytopes [40, 41] finding 1185 polytopes with $h^{1,1}=4 \leq \operatorname{Pic}_{X_{4}} \cdot{ }^{5}$ Out of these Calabi-Yau hypersurfaces we pick those that exhibit a reflexive section of co-dimension one since these are the models that, in the case of a compatible triangulation, admit a K3 fibration structure. In order to check for a reflexive section, we use the Package to Analyse Lattice Polytopes PALP [60] which can

\footnotetext{
${ }^{4}$ They also proposed to use models with intersecting $\mathrm{dP}_{8}$ that do not allow for a diagonal structure of the volume.

${ }^{5} \mathrm{Pic}_{X_{4}} \geq 4$ is only a necessary condition for inducing four primitive divisor classes on the three-fold from the toric ambient space. The reason is that a toric divisor, which corresponds to a real interior point of a maximal dimensional facet of the polytope, would not intersect the Calabi-Yau hypersurface.
} 
scan for such a section up to co-dimension three. Imposing this selection criterion we are left with only 650 polytopes.

After having a list of relevant K3-fibred Calabi-Yau three-folds, we seek for those that have a volume form of 'weak' Swiss-cheese type. To be more specific, we demand the presence of a rigid divisor $D$ which is a 'diagonal' del Pezzo (2.16) given that this is the particular kind of four-cycle that can support the non-perturbative effects needed to realise the LVS $[13,14]$. In order to check this property, we have to construct the toric varieties corresponding to the lattice polytopes and calculate the properties of the toric divisors intersecting the Calabi-Yau hypersurface.

The toric ambient space can be obtained by triangulating the polytope. Hence, in the case when the triangulation of a polytope is not unique, we find more than just one toric variety for the polytope. This may not necessarily result in different Calabi-Yau threefolds, and so, for simplicity, we will treat all these hypersurfaces as different varieties. We work out the triangulations using an extended version of PALP [61] which calculates also the Mori cone of the toric variety and, with the help of SINGULAR [62], the intersection ring of the toric divisors on the Calabi-Yau. With the intersection numbers at hands, we then obtain the topological numbers of the toric divisors on the hypersurface.

Imposing one by one the above constraints, we find 540 lattice polytopes with at least one del Pezzo surface and 228 with at least two. As we have already stressed above, a second toric del Pezzo divisor might be needed in order to realise chiral $D$-brane constructions within a LVS with stabilised closed string moduli. Finally, out of the 540 polytopes, 158 have at least one triangulation that admits a K3 fibration with a 'diagonal' del Pezzo. The list of these polytopes is given in appendix B.

In the next two sections we shall work out in detail some illustrative examples chosen from these 158 polytopes. We shall start from the case of simplicial ones where all the del Pezzo divisors are 'diagonal', and then move to discuss the case of non-simplicial polytopes with the interesting emergence of 'non-diagonal' del Pezzo four-cycles.

\section{$5 \quad$ K3 fibrations from simplicial polytopes}

The first class of examples that we consider, are Calabi-Yau hypersurfaces embedded in toric ambient spaces which are given by simplicial lattice polytopes. Here, simplicial means that the number of vertices of the polytope equals the dimension plus one. For the corresponding toric manifolds all but one divisor classes come from resolutions of singularities of the toric variety.

Out of the eleven four-dimensional, simplicial, reflexive lattice polytopes with $h^{1,1}\left(Y_{3}\right)=4$, ten have more than eight points, including the origin, such that $\mathrm{Pic}_{X_{4}} \geq 4$. However, only eight of them induce four primitive divisor classes from the toric four-fold on the Calabi-Yau hypersurface. Moreover, only six of them have a reflexive section of co-dimension one and five a triangulation which respects the fibration structure. The corresponding spaces have all the same structure.

The Calabi-Yau $\mathbb{P}_{[1,1,2,2,2]}^{4}(8) / \mathbb{Z}_{2}: 01100 \mathrm{~T} 1$, where $\mathrm{T} 1$ stands for the 'first' triangulation, will be the archetype example that we shall analyse in detail below. In this case we shall be able to find a basis in which the intersection polynomial com- 
pletely diagonalises. The other Calabi-Yau manifolds of this type are the three further triangulations of $\mathbb{P}_{[1,1,2,2,2]}(8) / \mathbb{Z}_{2}: 01100$ with a $\mathrm{K} 3$ fibration structure, one triangulation of $\mathbb{P}_{[1,1,2,2,2]}(8) / \mathbb{Z}_{2}: 01111$, four triangulations of $\mathbb{P}_{[1,1,2,2,6]}(12) / \mathbb{Z}_{2}: 01100$, one triangulation of $\mathbb{P}_{[1,1,2,2,6]}(12) / \mathbb{Z}_{2}: 01111$, and one, out of the four, triangulation of $\mathbb{P}_{[1,1,2,2,6]}(12) / \mathbb{Z}_{2}: 10001$ that admits a K3 fibration. They all share very similar intersection structures, and so, like in the illustrative $\mathbb{P}_{[1,1,2,2,2]}^{4}(8) / \mathbb{Z}_{2}: 01100 \mathrm{~T} 1$ case, their volume can be completely diagonalised.

\subsection{The exemplar: $\mathbb{P}_{[1,1,2,2,2]}^{4}(8) / \mathbb{Z}_{2}: 01100$ T1}

The toric ambient space $\mathbb{P}_{[1,1,2,2,2]}^{4}(8) / \mathbb{Z}_{2}: 01100$ can be defined by homogeneous coordinates and their equivalence relations. The weight matrix for the resolved variety reads:

\begin{tabular}{|c|c|c|c|c|c|c|c||c|}
\hline$z_{1}$ & $z_{2}$ & $z_{3}$ & $z_{4}$ & $z_{5}$ & $z_{6}$ & $z_{7}$ & $z_{8}$ & $D_{\mathrm{H}}$ \\
\hline \hline 2 & 1 & 0 & 0 & 2 & 2 & 0 & 1 & 8 \\
\hline 1 & 0 & 0 & 0 & 2 & 2 & 2 & 1 & 8 \\
\hline 1 & 1 & 0 & 2 & 2 & 2 & 0 & 0 & 8 \\
\hline 1 & 0 & 1 & 0 & 1 & 1 & 0 & 0 & 4 \\
\hline
\end{tabular}

Here, the $z_{i}$ are the homogeneous coordinates and $D_{\mathrm{H}}$ stands for the multi-degree of the hypersurface (divisor) which, in the Calabi-Yau case, is just the sum of the weights in a row. The surface of the reflexive lattice polytope encoding this weights exhibits several maximal triangulations. Given that we may construct a fan from each triangulation, we obtain more than just one toric variety for this weight matrix. In the following, we stick to the 'first' triangulation, T1, out of the four for this polytope. The triangulation and the fan, respectively, can be encoded in the Stanley-Reisner (SR) ideal, for which we obtain:

$$
\mathrm{SR}=\left\{z_{1} z_{8}, z_{2} z_{8}, z_{1} z_{2}, z_{1} z_{3}, z_{7} z_{8}, z_{3} z_{7}, z_{2} z_{6} z_{4} z_{5}, z_{3} z_{4} z_{5} z_{6}, z_{4} z_{5} z_{6} z_{7}\right\}
$$

The SR-ideal is the set of homogeneous coordinates that must not vanish simultaneously. From this and the linear equivalence relations given by the weight matrix we calculate the triple intersection numbers for a basis of divisor classes of the toric variety on the CalabiYau. Choosing the divisors $D_{i}=\left\{z_{i}=0\right\}, i=1, \ldots, 4$ as a basis, ${ }^{6}$ their triple intersections can be written in terms of the following polynomial:

$$
\begin{aligned}
I_{3}= & -24 D_{4}^{3}-8 D_{3}^{2} D_{4}-4 D_{2} D_{4}^{2}+4 D_{2} D_{3} D_{4}-2 D_{2} D_{3}^{2}-4 D_{2}^{2} D_{4} \\
& +2 D_{2}^{2} D_{3}-2 D_{2}^{3}+4 D_{1} D_{4}^{2} .
\end{aligned}
$$

The prefactors denote the intersection numbers of the respective divisors. Moreover, the generators $\mathcal{C}_{i}$ of the Mori cone, i.e. the cone of effective curves, of the toric variety are:

$$
\int_{\mathcal{C}_{i}} D_{j}=\left(\begin{array}{cccc}
1 & 1 & 0 & 0 \\
0 & -1 & 1 & -1 \\
0 & 1 & -2 & 0 \\
0 & 0 & 1 & 2
\end{array}\right) .
$$

\footnotetext{
${ }^{6}$ For ease of read, throughout all the paper we arranged the homogeneous coordinates in such a way that the first four are the (relevant) basis even if in general this is not the case.
} 
The Kähler form can be expanded in the previous basis as:

$$
J=\sum_{i=1}^{4} t_{i} D_{i},
$$

where we wrote down the sum explicitly and lowered the index of the $t$ 's for ease of read. It should however be understood that the $t$ 's and the $\tau$ 's are dual variables. We shall however perform a change of basis expanding $J$ in terms of the generators $\Gamma$ 's of the Kähler cone, i.e. the dual to the Mori cone, which in this basis becomes very simple:

$$
J=\sum_{i=1}^{4} r_{i} \Gamma_{i} \quad \text { with } \quad r_{i}>0 .
$$

The generators of the Kähler cone look like:

$$
\begin{array}{ll}
\Gamma_{1}=D_{1}, & \Gamma_{2}=4 D_{1}-4 D_{2}-2 D_{3}+D_{4}, \\
\Gamma_{3}=3 D_{1}-3 D_{2}-2 D_{3}+D_{4}, & \Gamma_{4}=2 D_{1}-2 D_{2}-1 D_{3}+D_{4} .
\end{array}
$$

With the Kähler form we can calculate the volumes of the divisors $D_{i}, i=1, \ldots, 4$ which turn out to be:

$$
\begin{array}{llrl}
\tau_{1} & =\frac{1}{2} \int_{D_{1}} J \wedge J=2\left(r_{2}+r_{3}+r_{4}\right)^{2}, & \tau_{2}=\tau_{1}-\left(r_{3}+r_{4}\right)^{2}, \\
\tau_{3}=\tau_{1}-\tau_{2}-r_{4}^{2}, & \tau_{4}=2\left(r_{1} \sqrt{2 \tau_{1}}+2 \tau_{2}+\tau_{3}-\tau_{1}\right) .
\end{array}
$$

The volume of the Calabi-Yau manifold is given by:

$$
\begin{aligned}
\mathcal{V} & =\frac{1}{6} \int_{Y_{3}} J \wedge J \wedge J \\
& =\left[r_{1}+\frac{2}{3}\left(r_{2}+r_{3}+r_{4}\right)\right] 2\left(r_{2}+r_{3}+r_{4}\right)^{2}-\frac{1}{3}\left(r_{3}+r_{4}\right)^{3}-\frac{1}{3} r_{4}^{3} \\
& =\frac{\sqrt{\tau_{1}}}{6 \sqrt{2}}\left(10 \tau_{1}-12 \tau_{2}-6 \tau_{3}+3 \tau_{4}\right)-\frac{1}{3}\left(\tau_{1}-\tau_{2}-\tau_{3}\right)^{3 / 2}-\frac{1}{3}\left(\tau_{1}-\tau_{2}\right)^{3 / 2} .
\end{aligned}
$$

The form of the volume (5.9) suggests that we can find a 'diagonal' basis for the divisor classes. Following the algorithm presented in section 2.2 , we define: ${ }^{7}$

$$
\begin{array}{ll}
D_{a}:=D_{1}=: D_{K 3}, & D_{b}:=10 D_{1}-12 D_{2}-6 D_{3}+3 D_{4}, \\
D_{c}:=D_{1}-D_{2}-D_{3}=D_{8}, & D_{d}:=D_{1}-D_{2} .
\end{array}
$$

In this basis, the intersection polynomial takes the form:

$$
I_{3}=36 D_{a} D_{b}^{2}+2 D_{c}^{3}+2 D_{d}^{3},
$$

showing that (5.9) is indeed a diagonal basis. The volumes of the diagonal divisors are:

$$
\begin{array}{ll}
\tau_{a}=\tau_{1}=2\left(r_{2}+r_{3}+r_{4}\right)^{2}, & \tau_{b}=10 \tau_{1}-12 \tau_{2}-6 \tau_{3}+3 \tau_{4}=\left[r_{1}+\frac{2}{3}\left(r_{2}+r_{3}+r_{4}\right)\right] 6 \sqrt{2 \tau_{1}} \\
\tau_{c}=\tau_{1}-\tau_{2}-\tau_{3}=r_{4}^{2}, & \tau_{d}=\tau_{1}-\tau_{2}=\left(r_{3}+r_{4}\right)^{2} .
\end{array}
$$

\footnotetext{
${ }^{7}$ The definition of $D_{K 3}$ will become clearer later on.
} 
Finally, we can rewrite the Calabi-Yau volume in terms of the above expressions:

$$
\mathcal{V}=\alpha\left(\tau_{b} \sqrt{\tau_{a}}-\beta \tau_{c}^{3 / 2}-\beta \tau_{d}^{3 / 2}\right)
$$

where $\alpha=1 /(6 \sqrt{2})$ and $\beta=2 \sqrt{2} / 3$. We point out that we managed to obtain a K3fibred Calabi-Yau three-fold of 'strong' Swiss-cheese type that is of the same form of the compactification manifolds used in $[23,24]$ for very promising cosmological applications. Moreover, in the limit of small $\tau_{c}$ and $\tau_{d}$ (for small $r_{3}$ and $r_{4}$ ), the overall volume is controlled by the two large cycles $\tau_{a}$ and $\tau_{b}$ :

$$
\mathcal{V} \simeq \alpha \tau_{b} \sqrt{\tau_{a}} \simeq r_{1} \tau_{1}+\frac{\sqrt{2}}{3} \tau_{1}^{3 / 2}
$$

where, as we shall see in the next section, $\tau_{a}=\tau_{1}$ gives the volume of the K3 fibre while $r_{1}$ is the volume of the $\mathbb{P}^{1}$ base of the fibration which is embedded into the Calabi-Yau by one of the four $\mathbb{P}^{1}$ 's of $D_{4} \cap D_{7}$.

It is interesting to notice that in the large volume limit $r_{1} \gg r_{2} \Leftrightarrow r_{1} \gg \sqrt{\tau_{1}}$, the characteristic size of the two-dimensional base is much bigger than the size of the four dimensions of the fibre. This anisotropic shape was used in [28, 29] for very interesting phenomenological applications.

\subsubsection{Divisor analysis}

From the weight matrix (5.1), we observe that there are five rigid divisors $D_{2}, D_{3}, D_{4}, D_{7}$ and $D_{8}$, i.e. the respective normal bundles do not have any non-vanishing global section on these divisors. A surface that is shrinkable or, in other words, that admits a blow-down map, should be an exceptional divisor which is always rigid. Therefore from now on we shall focus on these five rigid divisors and determine their geometry, trying to understand which of them is a del Pezzo surface. Notice that none of these divisors can be the K3 fibre, since the fibre must have a trivial normal bundle.

Using the adjunction formula in the form of [63]:

$$
c(S)=\frac{\prod_{i}^{8}\left(1+D_{i}\right)}{\left(1+D_{\mathrm{CY}}\right)(1+S)}=1+c_{1}(S)+c_{2}(S)+\ldots
$$

we can calculate the first and second Chern classes, $c_{1}$ and $c_{2}$, of the tangent bundles of a generic divisor $S$. From $c(S)$ we obtain the Todd classes, $\mathrm{Td}=1+\frac{1}{2} c_{1}+\frac{1}{12}\left(c_{1}^{2}+c_{2}\right)+\ldots$, that allow us to compute the Euler and holomorphic Euler characteristic of $S$ :

$$
\chi(S)=\sum_{i=0}^{2 \operatorname{dim}(S)}(-1)^{i} b^{i}=\int_{S} c_{\operatorname{dim}(S)}(S) \quad \text { and } \quad \chi_{h}(S)=\sum_{i=0}^{\operatorname{dim}(S)}(-1)^{i} h^{i, 0}=\int_{S} \operatorname{Td}(S)
$$

with $\operatorname{dim}(S)$ denoting the complex dimension of $S$. The considered divisors have the 
following intersection structures with the other divisors:

\begin{tabular}{|c|c|c|c|c|c|c|c|c|}
\hline & $D_{1}$ & $D_{2}$ & $D_{3}$ & $D_{4}$ & $D_{5}$ & $D_{6}$ & $D_{7}$ & $D_{8}$ \\
\hline \hline$D_{2}^{2}$ & 0 & -2 & 2 & -4 & -2 & -2 & 0 & 0 \\
\hline$D_{3}^{2}$ & 0 & -2 & 0 & -8 & -4 & -4 & 0 & 2 \\
\hline$D_{4}^{2}$ & 4 & -4 & 0 & -24 & -8 & -8 & 0 & 8 \\
\hline$D_{7}^{2}$ & 4 & 4 & 0 & -8 & -8 & -8 & -16 & 0 \\
\hline$D_{8}^{2}$ & 0 & 0 & -2 & -4 & -2 & -2 & 0 & 2 \\
\hline
\end{tabular}

The divisor $D_{8}$ has triple self-intersection $D_{8}^{3}=2$ and otherwise only vanishing or negative entries in table (5.16). This translates into:

$$
\int_{\mathcal{C}=D_{i} \cap S} c_{1}(S)=\int_{Y_{3}} D_{i} \wedge S \wedge\left(-c_{1}\left(\mathcal{N}_{S \mid Y_{3}}\right)\right)=-S^{2} \cdot D_{i}>0 \quad \forall \mathcal{C}: D_{i} \neq S \wedge D_{i} \cap S \neq \emptyset,
$$

which is a necessary condition for $S$ to be a del Pezzo divisor. The Euler characteristic and holomorphic Euler number of this divisor are ten and one, respectively. These are the topological data of a $\mathrm{dP}_{7}$.

We can show algebraically that $D_{8}$ is a del Pezzo four-cycle by starting to analyse the toric divisor $D_{8},\left\{z_{8}=0\right\}$, without the hypersurface equation. In order to obtain its defining (three-dimensional) fan, we take the part of the (four-dimensional) fan that is made up by the maximal dimensional cones of the fan that contain the ray corresponding to $z_{8}$, and project it along this ray. The resulting space is $\mathbb{P}_{[1,2,1,1]}$ with $\left(z_{3}, z_{4}, z_{5}, z_{6}\right) \sim\left(\lambda z_{3}, \lambda^{2} z_{4}, \lambda z_{5}, \lambda z_{6}\right)$ if we keep the numbering as it was before the projection. Setting $z_{8}=0$ in the hypersurface and restricting to $D_{8}$ is equivalent to dropping all the monomials with $z_{8}$ and setting to one all the homogeneous coordinates $z_{j}$ that lie with $z_{8}$ in the SR-ideal, i.e. $z_{j} z_{8} \subset$ SR. In toric language this corresponds to taking a section through the dual M-lattice polytope. Therefore we obtain an equation of degree four in the homogeneous coordinates $z_{3}, z_{4}, z_{5}$, and $z_{6}$. Given that $\mathbb{P}_{[1,2,1,1]}(4)$ is a del Pezzo seven surface, we do not only know the topological numbers of the intersection of $D_{8}$ with the Calabi-Yau hypersurface but also its geometry. For the other rigid surfaces the intersection numbers (5.16) do not fulfill (5.17), implying that these divisors cannot be del Pezzo. Therefore we shall not further analyse them.

As a next step, we get to the K3 divisor. By computing tables like the one above for the rest of the divisors, one can easily find that $\int_{D_{1}} c_{1}\left(D_{1}\right) \wedge \imath^{*} D_{i}=-D_{1} \cdot D_{1} \cdot D_{i}=0$. Since we have also that $\int_{D_{1}} \imath^{*} c_{2}\left(Y_{3}\right)>0$, the theorem of [45] implies that this Calabi-Yau three-fold is a K3 fibration over $\mathbb{P}^{1}$ with typical fibre $D_{1}$. This can be checked by a calculation of the Euler and holomorphic Euler characteristics of $D_{1}$ which are 24 and two, as it must be for a K3 surface. Repeating the same analysis as in the case of $D_{8}$, we obtain $\mathbb{P}^{3}(4)$ for the intersection of $D_{1}$ with the hypersurface.

Furthermore, we can also make the projection $\pi$ of the fibration explicit. We will explain the details of the derivation of this map for the next example and give here just the result:

$$
\pi: \quad\left[z_{1}: \ldots: z_{8}\right] \mapsto\left[z_{1}: z_{2} z_{3} z_{8}\right] \in \mathbb{P}^{1}
$$


where $\mathbb{P}^{1}$ is the base of the fibration. This fibration has also a section since the hypersurface equation becomes independent of $z_{1}, z_{2}, z_{3}$ and $z_{8}$ at the locus $D_{4} \cap D_{7}$. One can choose one of the four solutions of $z_{5}^{4}=\alpha z_{6}^{4}$, with some $\alpha \in \mathbb{C}$, as the embedding of the $\mathbb{P}^{1}$ base of the fibration.

Finally, we look at the shrinkability of these cycles recalling that, according to Grauter's criterion [64, 65], on a Calabi-Yau only del Pezzo four-cycles can be contracted to a point. Indeed, taking the limit $r_{4} \rightarrow 0 \Leftrightarrow D_{8} \rightarrow 0$ does not force any of the other cycles to shrink to zero size. The other rigid divisors are at most contractible to a curve, e.g. $D_{3}$ and $D_{7}$, without severely influencing the other cycles.

The other triangulations of this polytope which admit a K3 fibration, T2 and T4, can be related to T1 via flop transitions. For T2 the properties of $D_{7}$ and $D_{8}$ get exchanged with those of $D_{2}$ and $D_{4}$. On the other hand, the last triangulation T4 respects the symmetry of the polytope and treats $D_{2}, D_{8}$ and $D_{4}, D_{7}$ equivalently. In this case both $D_{2}$ and $D_{8}$ become a del Pezzo four-cycle.

\subsubsection{Orientifolding}

Due to the fact that type IIB compactifications give rise to $\mathcal{N}=1$ supersymmetry in four dimensions only if the internal manifold is a Calabi-Yau orientifold, in this section we shall study what happens to the geometry in the orientifold case.

Depending on the choice of the involution, we have to fix several complex structure moduli so that the Calabi-Yau hypersurface becomes invariant under the involution. For an involution $z_{i} \leftrightarrow-z_{i}$, the transformed coordinate must have even powers in the hypersurface equation, and so all the monomials where the coordinate shows up with odd powers are set to zero. Therefore we do not consider generic Calabi-Yau hypersurfaces, but only those that lie on a particular subspace of the complex structure moduli space.

For the Calabi-Yau three-fold under consideration, we shall consider the $\mathbb{Z}_{2}$-involution $z_{5} \leftrightarrow-z_{5}$. Following the algorithm presented in [42], we obtain the following fixed point set of the toric four-fold:

$$
\left.\{\text { Fixed }\}\right|_{z_{5} \leftrightarrow-z_{5}}=\left\{D_{5}\right\} .
$$

Therefore we end up with one $O 7$-plane at the intersection of the Calabi-Yau hypersurface with the toric divisor $D_{5}$.

\section{$6 \quad$ K3 fibrations from non-simplicial polytopes}

In this section we shall present two illustrative examples from non-simplicial polytopes. The first case admits two del Pezzo divisors: one 'diagonal' to support the non-perturbative effects that lead to moduli stabilisation, and one 'non-diagonal' for chiral $D$-brane constructions in the geometric regime. On the other hand, the second case admits just one 'diagonal' del Pezzo four-cycle but it features an interesting F-theory uplift in terms of an elliptically fibred Calabi-Yau four-fold. Another reason why we chose these models is because, besides featuring at least one 'diagonal' del Pezzo divisor, they also have one homogeneous coordinate with a high multi-degree. This is an advantage in the case of type 
IIB model building on Calabi-Yau orientifolds where there is a generic need of $O$-planes with large divisor classes.

\subsection{A K3 fibration with two del Pezzo divisors}

Like in section 5.1, we give the toric ambient variety by its weight matrix:

\begin{tabular}{|c|c|c|c|c|c|c|c||c|}
\hline$z_{1}$ & $z_{2}$ & $z_{3}$ & $z_{4}$ & $z_{5}$ & $z_{6}$ & $z_{7}$ & $z_{8}$ & $D_{\mathrm{H}}$ \\
\hline \hline 1 & 1 & 0 & 0 & 0 & 4 & 1 & 1 & 8 \\
\hline 0 & 1 & 0 & 0 & 1 & 6 & 2 & 2 & 12 \\
\hline 0 & 0 & 1 & 0 & 0 & 2 & 1 & 1 & 5 \\
\hline 0 & 0 & 0 & 1 & 0 & 3 & 1 & 1 & 6 \\
\hline
\end{tabular}

and its Stanley-Reisner ideal:

$$
\mathrm{SR}=\left\{z_{1} z_{2}, z_{2} z_{5}, z_{1} z_{3}, z_{1} z_{4}, z_{4} z_{6}, z_{3} z_{7} z_{8}, z_{5} z_{6} z_{7} z_{8}\right\} .
$$

Choosing the divisors $D_{i}=\left\{z_{i}=0\right\}, i=1, \ldots, 4$ as a basis, we obtain the following intersection structure on the Calabi-Yau hypersurface:

$$
I_{3}=2 D_{1}^{3}-2 D_{2} D_{3}^{2}+2 D_{3}^{3}+3 D_{2} D_{3} D_{4}-2 D_{2} D_{4}^{2}-6 D_{3} D_{4}^{2}+8 D_{4}^{3} .
$$

Note that in this basis the divisor $D_{1}$ has a manifest diagonal structure. The generators $\mathcal{C}_{j}$ of the Mori cone of the four-dimensional toric variety defined via (6.1) and (6.2), are:

$$
\int_{\mathcal{C}_{j}} D_{i}=\left(\begin{array}{cccc}
-1 & 0 & 0 & 0 \\
3 & 0 & 0 & 2 \\
0 & 1 & 0 & -2 \\
0 & 0 & -1 & 1 \\
0 & 0 & 3 & -2
\end{array}\right)=: \mathcal{M}_{j i}
$$

showing explicitly that the polytope is non-simplicial since $j$ runs from one to five instead of four. We proceed as in section 5.1 and expand the Kähler form in terms of the generators Г's of the Kähler cone which are dual to (6.4):

$$
J=\sum_{j=1}^{5} r_{j} \Gamma_{j} \quad \text { with } \quad r_{j}>0,
$$

and:

$$
\begin{array}{ll}
\Gamma_{1}=D_{2}, \quad \Gamma_{2}=2 D_{2}+D_{3}+D_{4}, & \Gamma_{3}=-2 D_{1}+6 D_{2}+2 D_{3}+3 D_{4} \\
\Gamma_{4}=-2 D_{1}+6 D_{2}+3 D_{3}+3 D_{4}, & \Gamma_{5}=6 D_{2}+2 D_{3}+3 D_{4} .
\end{array}
$$

The volumes of the divisors $D_{i}, i=1, \ldots, 4$, expressed in terms of the Kähler parameters $r_{j}$, turn out to be:

$$
\begin{aligned}
& \tau_{1}=4\left(r_{3}+r_{4}\right)^{2}, \quad \tau_{4}=r_{1}\left(r_{2}+3 r_{4}\right), \\
& \tau_{2}=\left(r_{2}+r_{3}+3 r_{4}+r_{5}\right)\left(r_{2}+5 r_{3}+3 r_{4}+5 r_{5}\right)-\left(r_{2}+3 r_{4}\right)\left(r_{3}+r_{5}\right), \\
& \tau_{3}=r_{1}\left(r_{2}+5 r_{3}+3 r_{4}+5 r_{5}\right)+\left(r_{3}+r_{5}\right)\left(2 r_{2}+7 r_{3}+6 r_{4}+7 r_{5}\right),
\end{aligned}
$$


while the Calabi-Yau volume reads:

$$
\begin{aligned}
\mathcal{V}= & r_{1} \tau_{2}+\frac{2}{3} r_{2}^{3}-\frac{1}{3} \tau_{1}^{3 / 2} \\
& +\frac{1}{3} r_{3}\left[r_{2}\left(18 r_{2}+51 r_{3}+108 r_{4}+102 r_{5}\right)+r_{3}\left(28 r_{3}+105 r_{4}+132 r_{5}\right)+r_{4}\left(114 r_{4}+306 r_{5}\right)\right] \\
& +\frac{1}{3} r_{4}\left[r_{2}\left(18 r_{2}+54 r_{4}+108 r_{5}\right)+r_{4}\left(38 r_{4}+162 r_{5}\right)+1710 r_{5}^{2}\right] \\
& +2 r_{5}\left[r_{2}\left(3 r_{2}+51 r_{5}\right)+44 r_{5}^{2}\right] .
\end{aligned}
$$

Given that we are dealing with a non-simplicial Kähler cone, the $\Gamma$ 's are linearly dependent even if we have only four Kähler moduli. However, in order to be able to write down the effective field theory, we need to know the independent degrees of freedom which become manifest if we expand the Kähler form in terms of the initial basis $D_{i}, i=1, \ldots, 4$ :

$$
J=\sum_{i=1}^{4} t_{i} D_{i}, \quad \text { with } \quad \sum_{i=1}^{4} \mathcal{M}_{j i} t_{i}>0 .
$$

The volume of the basis divisor and the Calabi-Yau three-fold as a function of the four linear independent $t$ 's looks like:

$$
\begin{array}{ll}
\tau_{1}=t_{1}^{2}, & \tau_{2}=5 t_{3} t_{4}-\left(t_{3}+t_{4}\right)^{2}, \\
\tau_{3}=t_{3}^{2}-3 t_{4}^{2}+t_{2}\left(3 t_{4}-2 t_{3}\right), & \tau_{4}=\left(t_{2}-2 t_{4}\right)\left(3 t_{3}-2 t_{4}\right),
\end{array}
$$

and:

$$
\begin{aligned}
\mathcal{V} & =t_{2}\left[5 t_{3} t_{4}-\left(t_{3}+t_{4}\right)^{2}\right]+\frac{1}{3}\left[t_{3}^{3}-9 t_{3} t_{4}^{2}+4 t_{4}^{3}\right]+\frac{t_{1}^{3}}{3} \\
& =\left(t_{2}-2 t_{4}\right) \tau_{2}-\frac{1}{3}\left(t_{3}-2 t_{4}\right)\left(\tau_{2}+t_{3} t_{4}\right)-\frac{1}{3} \tau_{1}^{3 / 2} .
\end{aligned}
$$

Contrary to the previous simplicial example, we have not been able to write the volume just in terms of Kähler moduli $\tau_{i}$. Thus we did not manage to diagonalise the volume form completely, even if the modulus $\tau_{1}$ is manifestly diagonal.

As we shall explain in the next section, this manifolds admits two del Pezzo divisors: $D_{1}$ which is a $\mathrm{dP}_{7}$ and $D_{4}$ which is a $\mathrm{dP}_{1}$. However only $D_{1}$ turns out to be a 'diagonal' del Pezzo, while $D_{4}$ is a 'non-diagonal' del Pezzo four-cycle which could be very useful for chiral $D$-brane model building.

Let us now show that we can indeed write down the volume form just in terms of the $\tau$ 's in the anisotropic large volume limit used in [28, 29]. We start by taking the limit of small $\tau_{1}$. As can be seen from (6.8), this is equivalent to sending $r_{3} \rightarrow 0$ and $r_{4} \rightarrow 0$. In this limit, the divisor volumes become:

$$
\tau_{1} \rightarrow 0, \quad \tau_{4} \rightarrow r_{1} r_{2}, \quad \tau_{2} \rightarrow r_{2}^{2}+5 r_{2} r_{5}+5 r_{5}^{2}, \quad \tau_{3} \rightarrow r_{1}\left(r_{2}+5 r_{5}\right)+2 r_{2} r_{5}+7 r_{5}^{2},
$$

while the Calabi-Yau volume reduces to:

$$
\mathcal{V} \rightarrow r_{1} \tau_{2}+\frac{2}{3} r_{2}^{3}+6 r_{2}^{2} r_{5}+102 r_{2} r_{5}^{2}+88 r_{5}^{3} .
$$

As we shall see in the next section, the volume of the K3 fibre is given by $\tau_{2}$ while the volume of the $\mathbb{P}^{1}$ base of the fibration is controlled by the Kähler parameter $r_{1}$. Therefore 
the anisotropic limit $r_{1} \gg \sqrt{\tau_{2}}$ corresponds to $r_{1} \gg r_{2} \sim r_{5}$. In this new limit the expressions (6.12) and (6.13) further simplify to:

$$
\tau_{1} \rightarrow 0, \quad \tau_{4} \rightarrow r_{1} r_{2}, \quad \tau_{2} \rightarrow r_{2}^{2}+5 r_{2} r_{5}+5 r_{5}^{2}, \quad \tau_{3} \rightarrow r_{1}\left(r_{2}+5 r_{5}\right), \quad \mathcal{V} \rightarrow r_{1} \tau_{2} .
$$

We can now invert the expression of the $r$ 's in terms of the $\tau$ 's obtaining:

$$
r_{1}=\frac{\sqrt{\tau_{3}^{2}+3 \tau_{3} \tau_{4}+\tau_{4}^{2}}}{\sqrt{5 \tau_{2}}}, \quad r_{2}=\frac{\tau_{4} \sqrt{5 \tau_{2}}}{\sqrt{\tau_{3}^{2}+3 \tau_{3} \tau_{4}+\tau_{4}^{2}}}, \quad r_{5}=\frac{\left(\tau_{3}-\tau_{4}\right) \sqrt{\tau_{2}}}{\sqrt{5} \sqrt{\tau_{3}^{2}+3 \tau_{3} \tau_{4}+\tau_{4}^{2}}}
$$

Hence, the Calabi-Yau volume in the limit $r_{1} \gg r_{2} \sim r_{5} \gg r_{3} \sim r_{4}>0$ takes the final form:

$$
\mathcal{V}=\sqrt{\frac{\tau_{2}}{5}}\left(\tau_{3}^{2}+3 \tau_{3} \tau_{4}+\tau_{4}^{2}\right)^{1 / 2}-\frac{1}{3} \tau_{1}^{3 / 2}
$$

\subsubsection{Divisor analysis}

In this section we shall analyse the topology of the divisors of the Calabi-Yau three-fold under consideration starting from the rigid divisors and then turning to the study of the fibration structure.

By inspecting the weight matrix (6.1), we notice that this manifold has four rigid divisors: $D_{1}, D_{3}, D_{4}$ and $D_{5}$. However only $D_{1}$ and $D_{4}$ fulfill the necessary condition (5.17) to be a del Pezzo divisor. In fact, the topological indices of these surfaces indicate that they are a $\mathrm{dP}_{7}$ and $\mathrm{dP}_{1}$, respectively. In order to make this more explicit, we proceed as above and project the parts of the fans that have the respective ray as a subset along this ray. From the analysis of the so obtained fans, together with the transformed hypersurfaces, ${ }^{8}$ we get for the two surfaces:

$$
\begin{aligned}
& D_{1}: \begin{array}{|c|c|c|c||c|c|}
\hline z_{5} & z_{6} & z_{7} & z_{8} & \left.D_{\mathrm{H}}\right|_{D_{1}} \\
\hline 1 & 2 & 1 & 1 & 4 & 4
\end{array} \quad \text { with }\left.\quad \mathrm{SR}\right|_{D_{1}}=\left\{z_{5} z_{6} z_{7} z_{8}\right\} \text {, and } \\
& D_{4}: \\
& \begin{array}{|c|c|c|c|c||c|c|c|}
z_{2} & z_{3} & z_{5} & z_{7} & z_{8} & \left.D_{\mathrm{H}}\right|_{D_{4}} \\
\hline 0 & 3 & 0 & 1 & 1 & 3 \\
\hline 1 & 0 & 1 & 0 & 0 & 0 \\
\end{array}
\end{aligned}
$$

Hence $D_{1}$ is a del Pezzo seven surface while $D_{4}$ is a $\mathbb{P}^{1} \times \mathbb{P}^{1}$.

In order to find the K3 fibre, we search, by means of PALP, for all reflexive sections of co-dimension one of the N-lattice polytope which implies that the dual M-lattice polytope has a reflexive projection. Given that the M-lattice polytope encodes the information about the monomials of the hypersurface equation, the projection can be interpreted as the original equation with the homogeneous coordinates, not on the section, set to some generic value. The reflexivity then assures that the equation, in the homogeneous coordinates lying on the section, is a Calabi-Yau two-fold, i.e. a K3 surface. By using the theorem of [45] we can then easily see whether this is also a fibration.

In order to make this more explicit in terms of toric language, we need that the projection along the section of the polytope maps the fan of the toric four-fold into a valid

\footnotetext{
${ }^{8}$ Note again that the homogeneous coordinates not intersecting the respective divisor are omitted.
} 
fan. This toric homomorphism induces then the projection of the fibration. The polytope under consideration has one section of this kind and the only points that do not lie on this plane are the ones corresponding to $z_{1}, z_{2}$ and $z_{5}$. From the projection along this plane we obtain the two vectors of the $\mathbb{P}^{1}$ where $z_{1}$ and $z_{5}$ are mapped to the same point. Hence the homogeneous coordinates of the base $\mathbb{P}^{1}$ are $\zeta_{1}=z_{2}$ and $\zeta_{2}=z_{1} z_{5}$. In order to see that this projection is also a homomorphism between the fans, we inspect the SR-ideals of the two toric varieties. We have to check whether $\mathrm{SR}_{\mathbb{P}^{1}}=\left\{\zeta_{1} \zeta_{2}\right\} \simeq\left\{z_{1} z_{2}, z_{2} z_{5}\right\}$ is a subset of the SR-ideal (6.2) of the original toric four-fold. Given that this is indeed the case, we have a globally defined projection of the four-dimensional toric variety to $\mathbb{P}^{1}$. The corresponding three-dimensional toric fibre:

\begin{tabular}{|c|c|c|c|c||c|}
\hline$z_{3}$ & $z_{4}$ & $z_{6}$ & $z_{7}$ & $z_{8}$ & $\left.D_{\mathrm{H}}\right|_{\text {fibre }}$ \\
\hline 1 & 0 & 2 & 1 & 1 & 5 \\
\hline 0 & 1 & 3 & 1 & 1 & 6 \\
\hline
\end{tabular}$\quad$ with $\left.\quad \mathrm{SR}\right|_{\text {fibre }}=\left\{z_{4} z_{6}, z_{3} z_{7} z_{8}\right\}$,

degenerates at the point $\zeta_{2}=0$ into two toric divisors, $D_{1}$ and $D_{5}$, while at $\zeta_{1}=0$ we have the generic fibre. Thus, together with the hypersurface equation, we obtain a generic K3 fibre over this point. For this fibration we have also global sections. Given that the three $\mathbb{P}^{1}$ 's turn out to be $\mathcal{C}_{3 \mathbb{P}^{1}}=D_{3} \cap D_{4}$, the volume of the base is:

$$
\operatorname{Vol}(\text { base })=\frac{1}{3} \operatorname{Vol}\left(\mathcal{C}_{3 \mathbb{P}^{1}}\right)=\frac{1}{3} \int_{D_{3} \cap D_{4}} \imath^{*} J=\frac{1}{3} D_{3} \cdot D_{4} \cdot J=r_{1}=t_{2}-2 t_{4} .
$$

Let us now summarise the results of our divisor analysis:

\begin{tabular}{|c|c|c|c|}
\hline divisor classes & $D_{1}$ & $D_{2} \simeq D_{1}+D_{5}$ & $D_{4}$ \\
\hline variety & $\mathrm{dP}_{7}$ & $\mathrm{~K} 3$ & $\mathbb{P}^{1} \times \mathbb{P}^{1}$ \\
\hline volume & $4\left(r_{3}+r_{4}\right)^{2}$ & $\left(\begin{array}{c}\left(r_{2}+r_{3}+3 r_{4}+r_{5}\right)\left(r_{2}+5 r_{3}+3 r_{4}+5 r_{5}\right) \\
-\left(r_{2}+3 r_{4}\right)\left(r_{3}+r_{5}\right)\end{array}\right.$ & $r_{1}\left(r_{2}+3 r_{4}\right)$ \\
\hline
\end{tabular}

\subsubsection{Orientifolding}

We shall now compare the $\mathbb{Z}_{2}$-involutions $z_{6} \leftrightarrow-z_{6}$ and $z_{7} \leftrightarrow-z_{7}$. For the two cases, the generic hypersurface equation, expanded in the transformed coordinate, reduces to:

$$
\begin{aligned}
\left.P_{\mathrm{CY}}\right|_{z_{6} \leftrightarrow-z_{6}}= & z_{3} z_{6}^{2}+z_{4} P_{(8,12,5,5)}=0, \quad \text { and } \\
\left.P_{\mathrm{CY}}\right|_{z_{7} \leftrightarrow-z_{7}}= & z_{3} P_{(8,12,4,6)}+z_{8} P_{(7,10,4,5)}+z_{7}^{2}\left(z_{3} P_{(6,8,2,4)}+z_{8} P_{(5,6,2,3)}\right) \\
& +z_{7}^{4}\left(z_{3} P_{(4,4,0,2)}+z_{8} P_{(3,2,0,1)}\right),
\end{aligned}
$$

where the $P_{(i, j, k, l)}$ are generic polynomials, in the rest of the homogeneous coordinates, of the indicated class. The fixed point sets of the toric four-fold turns out to be:

$$
\begin{aligned}
& \left.\{\text { Fixed }\}\right|_{z_{6} \leftrightarrow-z_{6}}=\left\{D_{6}, D_{3} \cdot D_{4}, D_{4} \cdot D_{7} \cdot D_{8}, D_{1} \cdot D_{5} \cdot D_{7} \cdot D_{8}\right\} \quad \text { and } \\
& \left.\{\text { Fixed }\}\right|_{z_{7} \leftrightarrow-z_{7}}=\left\{D_{7}, D_{3} \cdot D_{8}, D_{1} \cdot D_{5} \cdot D_{8}\right\} .
\end{aligned}
$$


However, only $D_{6}$ and $D_{3} \cdot D_{4}$ in the first case, and $D_{7}, D_{3} \cdot D_{8}$, and $D_{1} \cdot D_{5} \cdot D_{8}$ in the second case, intersect the respective hypersurface. From (6.19), we observe that $D_{3} \cdot D_{4}$ and $D_{3} \cdot D_{8}$ induce a second $O 7$-plane on the corresponding Calabi-Yau hypersurfaces in addition to $D_{6}$ and $D_{7}$. Moreover, in the case of $z_{7} \leftrightarrow-z_{7}, D_{1} \cdot D_{5} \cdot D_{8}$ induces an $O 3$-plane.

We finally stress that in the orientifold case nothing severely happens for the divisors that we considered above. However, the toric divisor $D_{3}$ intersecting the hypersurface factorises into two parts in the orientifold case $z_{7} \leftrightarrow-z_{7}$, as can be deduced from (6.19).

\subsection{A K3 fibration with one del Pezzo divisor}

The second non-simplicial example that we work out in detail is a K3 fibration which admits only one toric del Pezzo divisor. The fan of the toric four-fold is defined in terms of its weight matrix:

\begin{tabular}{|c|c|c|c|c|c|c|c||c|}
\hline$z_{1}$ & $z_{2}$ & $z_{3}$ & $z_{4}$ & $z_{5}$ & $z_{6}$ & $z_{7}$ & $z_{8}$ & $D_{\mathrm{H}}$ \\
\hline \hline 0 & 0 & 1 & 0 & 0 & 1 & 1 & 3 & 6 \\
\hline 1 & 2 & 0 & 0 & 1 & 1 & 0 & 5 & 10 \\
\hline 1 & 1 & 1 & 0 & 0 & 1 & 0 & 4 & 8 \\
\hline 0 & 0 & 0 & 1 & 0 & 0 & 1 & 2 & 4 \\
\hline
\end{tabular}

and its Stanley-Reisner ideal:

$$
\mathrm{SR}=\left\{z_{1} z_{2}, z_{3} z_{6}, z_{1} z_{3}, z_{5} z_{6} z_{7}, z_{2} z_{5} z_{6}, z_{2} z_{5} z_{8}, z_{4} z_{7} z_{8}, z_{1} z_{4} z_{8}\right\}
$$

With these data we can calculate the intersection structure on the Calabi-Yau hypersurface in terms of the basis divisors $D_{i}=\left\{z_{i}=0\right\}, i=1, \ldots, 4$ :

$$
I_{3}=D_{1}^{3}+2 D_{2} D_{3} D_{4}-2 D_{3}^{2} D_{4}-2 D_{2} D_{4}^{2}+2 D_{4}^{3} .
$$

The Mori cone generators of the toric four-fold given by (6.21) and (6.22) are:

$$
\int_{\mathcal{C}_{j}} D_{i}=\left(\begin{array}{cccc}
0 & 1 & -1 & 0 \\
1 & 0 & 0 & 2 \\
-2 & 0 & 0 & -3 \\
0 & -2 & 2 & 1 \\
2 & 2 & 0 & -1
\end{array}\right):=\mathcal{M}_{j i}
$$

while the dual generators to (6.24) look like:

$$
\begin{array}{ll}
\Gamma_{1}=D_{2}+D_{3}, \quad \Gamma_{2}=4 D_{2}+3 D_{3}+2 D_{4}-3 D_{1}, & \Gamma_{3}=4 D_{2}+4 D_{3}+2 D_{4}-3 D_{1}, \\
\Gamma_{4}=5 D_{2}+4 D_{3}+2 D_{4}-4 D_{1}, & \Gamma_{5}=5 D_{2}+5 D_{3}+2 D_{4}-4 D_{1} .
\end{array}
$$

The fact that we found five, instead of just four, $\Gamma$ 's shows that the Kähler cone is again non-simplicial. Expanding the Kähler form in terms of the $\Gamma$ 's as:

$$
J=\sum_{j=1}^{5} r_{j} \Gamma_{j} \quad \text { with } \quad r_{j}>0
$$


the divisor volumes turn out to be:

$$
\begin{aligned}
\tau_{1}= & \frac{1}{2}\left[3 r_{2}+3 r_{3}+4\left(r_{4}+r_{5}\right)\right]^{2}, \\
\tau_{2}= & 4\left(r_{2}+r_{3}+r_{4}+r_{5}\right)\left[r_{1}+2 r_{2}+3\left(r_{3}+r_{4}\right)+4 r_{5}\right], \\
\tau_{3}= & 4\left(r_{2}+r_{4}\right)\left(r_{2}+r_{3}+r_{4}+r_{5}\right), \\
\tau_{4}= & r_{1}\left[r_{1}+4 r_{2}+4 r_{3}+6\left(r_{4}+r_{5}\right)\right]+r_{2}\left(5 r_{2}+12 r_{3}+14 r_{4}+16 r_{5}\right) \\
& +2 r_{3}\left[3 r_{3}+8\left(r_{4}+r_{5}\right)\right]+2 r_{4}\left(5 r_{4}+11 r_{5}\right)+11 r_{5}^{2},
\end{aligned}
$$

whereas the volume of the Calabi-Yau manifold becomes:

$$
\begin{aligned}
\mathcal{V}= & \frac{1}{6}\left(r_{2}+r_{3}\right)\left(73 r_{2}^{2}+170 r_{2} r_{3}+85 r_{3}^{2}\right)+2\left(22 r_{2}^{2}+48 r_{2} r_{3}+25 r_{3}^{2}\right) r_{4} \\
& +4\left(13 r_{2}+14 r_{3}\right) r_{4}^{2}+20 r_{4}^{3}+2\left[24 r_{2}^{2}+25 r_{3}^{2}+58 r_{3} r_{4}+32 r_{4}^{2}\right. \\
& \left.+r_{2}\left(50 r_{3}+56 r_{4}\right)\right] r_{5}+2\left[29\left(r_{2}+r_{3}\right)+33 r_{4}\right] r_{5}^{2}+22 r_{5}^{3}+2 r_{1}^{2}\left(r_{2}+r_{3}+r_{4}+r_{5}\right) \\
& +4 r_{1}\left(r_{2}+r_{3}+r_{4}+r_{5}\right)\left[3 r_{2}+3 r_{3}+4\left(r_{4}+r_{5}\right)\right]+\frac{4}{3}\left(r_{2}+r_{3}+r_{4}+r_{5}\right)^{3} .
\end{aligned}
$$

As we pointed out in the previous section, in order to write down the four-dimensional effective field theory we need to expand the Kähler form in terms of the initial basis $D_{i}$, $i=1, \ldots, 4$ as:

$$
J=\sum_{i=1}^{4} t_{i} D_{i}, \quad \text { with } \quad \sum_{i=1}^{4} \mathcal{M}_{j i} t_{i}>0 .
$$

The volume of the basis divisor and the Calabi-Yau three-fold as a function of the four linear independent $t$ 's looks like:

$$
\begin{array}{ll}
\tau_{1}=\frac{1}{2} t_{1}^{2}, & \tau_{2}=t_{4}\left(2 t_{3}-t_{4}\right), \\
\tau_{3}=2 t_{4}\left(t_{2}-t_{3}\right), & \tau_{4}=\frac{3}{2} t_{4}^{2}-t_{3}^{2}+2 t_{2}\left(t_{3}-t_{4}\right),
\end{array}
$$

and:

$$
\begin{aligned}
\mathcal{V} & =\frac{1}{6}\left[3 t_{4}\left(4 t_{2} t_{3}-2 t_{3}^{2}-2 t_{2} t_{4}+t_{4}^{2}\right)+t_{1}^{3}\right] \\
& =t_{2} \tau_{2}+\frac{t_{4}}{2}\left(t_{4}^{2}-2 t_{3}^{2}\right)-\frac{\sqrt{2}}{3} \tau_{1}^{3 / 2}
\end{aligned}
$$

Similarly to the previous non-simplicial example, we have not been able to write the volume just in terms of Kähler moduli $\tau_{i}$. Thus we did not manage to diagonalise the volume even if the modulus $\tau_{1}$ is manifestly diagonal. As we shall see in the next section, the divisor $D_{1}$ is a del Pezzo eight surface.

Let us now show that, by taking the limit of small $\tau_{1}$, the volume simplifies considerably, and so it can easily be written just in terms of the $\tau$ 's. As can be seen from (6.27), shrinking $\tau_{1}$ corresponds to taking $r_{1} \gg r_{2} \sim r_{3} \sim r_{4} \sim r_{5} \sim r>0$. In this limit, the divisor volumes become:

$$
\tau_{1} \sim 98 r^{2}, \quad \tau_{2} \sim 16 r r_{1}, \quad \tau_{3} \sim 32 r^{2}, \quad \tau_{4} \sim r_{1}^{2} \quad \Rightarrow \quad \tau_{4} \gg \tau_{2} \gg \tau_{1} \gtrsim \tau_{3},
$$


and so we notice that also the four-cycle $\tau_{3}$ is forced to shrink. On the other hand, the two-cycle parameters take the form:

$$
\begin{aligned}
& t_{1}=-\left(3 r_{2}+3 r_{3}+4 r_{4}+4 r_{5}\right) \sim-14 r \\
& t_{2}=r_{1}+4 r_{2}+4 r_{3}+5 r_{4}+5 r_{5} \sim r_{1}+18 r \\
& t_{3}=r_{1}+3 r_{2}+4 r_{3}+4 r_{4}+5 r_{5} \sim r_{1}+16 r \\
& t_{4}=2 r_{2}+2 r_{3}+2 r_{4}+2 r_{5} \sim 8 r, \quad \Rightarrow \quad t_{2} \gtrsim t_{3} \gg\left|t_{1}\right| \gtrsim t_{4} .
\end{aligned}
$$

Therefore the relations (6.30) simplify to:

$$
\tau_{1} \sim \frac{1}{2} t_{1}^{2}, \quad \tau_{2} \sim 2 t_{3} t_{4}, \quad \tau_{3} \sim 2 t_{4}\left(t_{2}-t_{3}\right), \quad \tau_{4} \sim t_{3}\left(2 t_{2}-t_{3}\right),
$$

while the Calabi-Yau volume reduces to:

$$
\mathcal{V}=t_{3} t_{4}\left(2 t_{2}-t_{3}\right)+\frac{1}{6} t_{1}^{3}=\frac{\left(2 t_{2}-t_{3}\right)}{2} \tau_{2}-\frac{\sqrt{2}}{3} \tau_{1}^{3 / 2}=\frac{\sqrt{\tau_{4}}}{2}\left(\tau_{2}+2 \tau_{3}\right)-\frac{\sqrt{2}}{3} \tau_{1}^{3 / 2},
$$

where, as we shall see in the next section, the volume of the K3 fibre is given by $\tau_{2}$. We conclude this section by showing that this limit corresponds to the anisotropic limit of $[28,29]$ since:

$$
\frac{\left(2 t_{2}-t_{3}\right)}{2} \sim \frac{r_{1}}{2} \gg \sqrt{\tau_{2}} \sim 4 \sqrt{r r_{1}} .
$$

\subsubsection{Divisor analysis}

We now turn to the study of the topology of the divisors of the Calabi-Yau three-fold under consideration.

By looking at the weight matrix (6.21), we realise that the rigid divisors of the CalabiYau three-fold are $D_{1}, D_{3}, D_{4}, D_{5}$ and $D_{7}$. However, only $D_{1}$ is a del Pezzo. Its topological numbers, $\chi_{h}=1$ and $\chi=11$, indicate that this divisor is a $\mathrm{dP}_{8}$. In order to check this, we project the relevant part of the fan along $z_{1}$ obtaining:

$$
D_{1}: \begin{array}{|c|c|c|c|c||c|}
\hline z_{4} & z_{5} & z_{6} & z_{7} & z_{8} & \left.D_{\mathrm{H}}\right|_{D_{1}} \\
\hline 0 & 1 & 1 & 2 & 3 & 6 \\
\hline 1 & 0 & 0 & 1 & 2 & 4 \\
\hline
\end{array}
$$

which shows explicitly that $D_{1}$ is a del Pezzo eight surface.

In order to find the K3 fibration we look for the reflexive sections of the N-lattice polytope encoded in (6.21) finding that the polytope at hands admits just one section of this type. The vectors corresponding to the homogeneous coordinates $z_{1}, z_{2}$ and $z_{5}$ do not lie on this plane. The projection along the plane maps $z_{2}$ to $\zeta_{1}$ and $z_{1} z_{5}$ to $\zeta_{2}$, where $\zeta_{1}$ and $\zeta_{2}$ are the homogeneous coordinates of the $\mathbb{P}^{1}$. In this case the projection does not define a toric homomorphism because $\mathrm{SR}_{\mathbb{P}^{1}}=\left\{\zeta_{1} \zeta_{2}\right\} \simeq\left\{z_{1} z_{2}, z_{2} z_{5}\right\}$ is not a subset of (6.22). However, the Calabi-Yau hypersurface equation does not go through the points $z_{2}=z_{5}=0$. Hence we may remove from the fan all the cones that contain the two-cone $\left\langle v_{2} v_{5}\right\rangle$. Even if the toric four-fold of the new fan is not compact anymore, the hypersurface is the same. For the new toric four-fold, $z_{2} z_{5}$ is part of the SR-ideal, and so we obtain a 
valid projection. This is in agreement with the theorem of [45]. Over the point $\zeta_{1}=z_{2}=0$, we get a generic K3 fibre and at $\zeta_{2}=0$ the fibre becomes the sum of $D_{1}$ and $D_{5}$. We finally point out that for this fibration it is not possible to find a section that embeds the base into the Calabi-Yau.

Let us now summarise the results of our divisor analysis:

\begin{tabular}{|c|c|c|}
\hline divisor classes & $D_{1}$ & $D_{2} \simeq D_{1}+D_{5}$ \\
\hline variety & $\mathrm{dP}_{8}$ & $\mathrm{~K} 3$ \\
\hline volume & $\frac{1}{2}\left[3 r_{2}+3 r_{3}+4\left(r_{4}+r_{5}\right)\right]^{2}$ & $4\left(r_{2}+r_{3}+r_{4}+r_{5}\right)\left[r_{1}+2 r_{2}+3\left(r_{3}+r_{4}\right)+4 r_{5}\right]$ \\
\hline
\end{tabular}

\subsubsection{Orientifolding}

We again briefly study the orientifold case by comparing the two different involutions $z_{6} \leftrightarrow-z_{6}$ and $z_{8} \leftrightarrow-z_{8}$. For the fixed point sets of the ambient space, we obtain:

$$
\begin{aligned}
\left.\{\text { Fixed }\}\right|_{z_{6} \leftrightarrow-z_{6}}= & \left\{D_{6}, D_{3} \cdot D_{4} \cdot D_{8}, D_{1} \cdot D_{5} \cdot D_{8}, D_{2} \cdot D_{4} \cdot D_{5}, D_{3} \cdot D_{7} \cdot D_{8},\right. \\
& \left.D_{3} \cdot D_{4} \cdot D_{7}, D_{2} \cdot D_{5} \cdot D_{7}, D_{1} \cdot D_{4} \cdot D_{5} \cdot D_{7}\right\} \text { and } \\
\left.\{\text { Fixed }\}\right|_{z_{8} \leftrightarrow-z_{8}}= & \left\{D_{8}, D_{4} \cdot D_{7}, D_{1} \cdot D_{5} \cdot D_{6}, D_{2} \cdot D_{3} \cdot D_{5}\right\} .
\end{aligned}
$$

However only $D_{6}, D_{3} \cdot D_{4} \cdot D_{8}, D_{1} \cdot D_{5} \cdot D_{8}$ and $D_{3} \cdot D_{7} \cdot D_{8}$ in the first case, and $D_{8}$ and $D_{1} \cdot D_{5} \cdot D_{6}$ in the second case, intersect the respective invariant hypersurface. Thus we get in both cases at least one $O 3$-plane in addition to the respective $O 7$-plane. Regarding the Calabi-Yau hypersurface, also the K3 fibre and the del Pezzo eight surface become less generic even if, together with all other toric divisors, they do not degenerate.

We finally have a quick look at the F-theory up-lift in the case of an orientifold with $z_{8} \leftrightarrow-z_{8}$ involution. By looking at the weight matrix (6.21), we realise that this CalabiYau three-fold has a structure similar to the one analysed in [66]. The $\mathbb{Z}_{2}$-quotient of the Calabi-Yau is the toric three-fold of (6.21) with $z_{8}$ omitted. Furthermore, the Calabi-Yau four-fold is given by a hypersurface in:

\begin{tabular}{|c|c|c||c|c|c|c|c|c|c||c|}
\hline$y$ & $x$ & $z$ & $z_{1}$ & $z_{2}$ & $z_{3}$ & $z_{4}$ & $z_{5}$ & $z_{6}$ & $z_{7}$ & $\sum_{Y_{4}}$ \\
\hline \hline 3 & 2 & 1 & 0 & 0 & 0 & 0 & 0 & 0 & 0 & 6 \\
\hline \hline 9 & 6 & 0 & 0 & 0 & 1 & 0 & 0 & 1 & 1 & 6 \\
\hline 15 & 10 & 0 & 1 & 2 & 0 & 0 & 1 & 1 & 0 & 10 \\
\hline 12 & 8 & 0 & 1 & 1 & 1 & 0 & 0 & 1 & 0 & 8 \\
\hline 6 & 4 & 0 & 0 & 0 & 0 & 1 & 0 & 0 & 1 & 4 \\
\hline
\end{tabular}

The Hodge number $h^{1,1}\left(Y_{4}\right)$ increased by one compared to $h_{+}^{1,1}\left(Y_{3}\right)=h^{1,1}\left(Y_{3}\right)=4$ of the original three-fold. The Euler characteristic of $Y_{4}$ is 13416 which is in agreement with the D3-tadpole of a type IIB orientifold with one Whitney brane [66] that cancels the $D 7$-tadpole. We stress that in this evaluation we also included the contribution to the tadpole coming from O3-planes finding that the stringy Hodge number calculation of [67] does indeed take the contribution of O3-planes into account. This is in contrast to [68] where the Euler number calculation matches the tadpole only without the O3-planes. ${ }^{9}$

\footnotetext{
${ }^{9}$ We are grateful to Sven Krause for doing the four-fold calculations and observing the mismatch.
} 


\section{Conclusions}

In this paper we used the powerful tools of toric geometry to construct several explicit examples of K3 fibrations which can be used in the study of Calabi-Yau string compactifications.

Historically this kind of manifolds received a lot of attention due to the crucial rôle that they play in the investigation of string dualities [2-9]. Therefore many explicit examples of K3-fibred Calabi-Yau three-folds have been built as hypersurfaces embedded either in complex weighted projective spaces $[1,2,10]$ or, more generally, in toric varieties [11]. The present work extends these constructions to include the interesting case of K3 fibrations with the presence of additional del Pezzo divisors.

The main motivation for these constructions is the relatively recent discovery of new LARGE Volume type IIB vacua [13] for K3-fibred Calabi-Yau three-folds [14] which turn out to be very promising for several applications to cosmology [23-25, 27] and particle phenomenology [28, 29]. As derived in [14], two conditions for the existence of such minima at exponentially large value of the internal volume are a negative Euler number, i.e. $h^{1,2}>$ $h^{1,1}>1$, and the presence of at least one local blow-up mode which is needed to support the non-perturbative effects that lead to moduli stabilisation by their interplay with $\alpha^{\prime}$ and $g_{s}$ corrections. However, before this paper, no explicit examples of K3 fibrations with additional blow-up modes were known in the literature.

Given that the final goal is not just to achieve moduli stabilisation but also to realise a chiral MSSM or GUT-like local construction with intersecting $D$-branes wrapped around internal divisors, we looked also for a second rigid divisor which could support chiral matter.

However, before performing our Calabi-Yau search, we gave a detailed description of the procedure to diagonalise the volume form and then we discussed briefly the interplay between a diagonal structure of the Calabi-Yau volume and the supersymmetric requirement of having vanishing Fayet-Iliopoulos terms. This discussion allowed us to classify the blow-up cycles in three different types:

i) 'Diagonal' del Pezzo divisors (with only their triple self intersection number nonvanishing, implying that they appear in the volume form in a completely diagonal manner) which are natural candidates for supporting the non-perturbative effects of the LVS or for leading to quiver GUT-like constructions due to their shrinking induced by the $D$-terms.

ii) 'Non-diagonal' del Pezzo divisors (with non-zero intersections with different divisors in any basis, implying that they do not appear in the Calabi-Yau volume in a completely diagonal way) which are suitable to support GUT constructions with magnetised branes wrapping cycles stabilised in the geometric regime since they are rigid and, above all, in this 'non-diagonal' case, the vanishing $D$-term condition might not force the shrinking of any cycle.

iii) Rigid but not del Pezzo divisors (not contractible to a point) which, similarly to the 'non-diagonal' del Pezzo divisors, cannot be used to support the non-perturbative effects in the LVS, but are natural candidates for GUT model building in the geometric regime. 
After this classification of blow-up cycles, we went through the existing list of four dimensional reflexive lattice polytopes [40, 41] searching for K3 fibrations with four Kähler moduli where at least one of them is a 'diagonal' del Pezzo divisor. The final result of our search consists of 158 lattice polytopes corresponding to toric ambient varieties which admit a Calabi-Yau hypersurface with the required features. We then performed a detailed analysis of the topology of some illustrative examples derived from both simplicial and non-simplicial polytopes. Interestingly enough, we found the presence of 'non-diagonal' rigid divisors only in the case of non-simplicial polytopes.

We point out that this paper represents just a first step towards the final goal of bringing together the existing scenarios for moduli stabilisation and chiral $D$-brane model building. The next step would be to use these compact Calabi-Yau three-folds to try to achieve full closed string moduli stabilisation in a globally consistent model (tadpole and Freed-Witten anomaly free) with an explicit brane set-up and a particular choice of worldvolume fluxes that lead to the local presence of chirality. We will discuss these matters in more detail in a forthcoming companion article [69].

We finally stress that the main motivation behind this paper has been to provide a more rigorous mathematical foundation to the interesting LVS. However the several explicit realisations of K3-fibrations with additional del Pezzo divisors that we presented in this paper could be very useful also for other completely different model building scenarios in string compactifications.

\section{Acknowledgments}

We would like to thank Sven Krause, Sven Krippendorf, Fernando Quevedo, Roberto Valandro, Nils-Ole Walliser, and Timo Weigand for useful discussions and especially Andrés Collinucci for initial collaboration on this project. We thank the Abdus Salam International Centre for Theoretical Physics for hospitality during the development of part of this work. The work of CM was supported by the DFG through TRR33 "The Dark Universe".

\section{A Two K3 fibrations with an F-theory uplift}

As a result of our search, 58 of the $158 \mathrm{~K} 3$-fibred Calabi-Yau manifolds with at least one 'diagonal' del Pezzo divisor that we found, feature an interesting F-theory uplift in terms of an elliptically fibred Calabi-Yau four-fold. In this appendix we shall present two simplicial examples of these K3 fibrations with such an uplift.

\section{A.1 $\mathbb{P}_{[1,1,1,1,4]}^{4}(8)$ with three blow-ups}

In order to define the fan of the toric four-fold, we give its weight matrix:

\begin{tabular}{|c|c|c|c|c|c|c|c||c|}
\hline$z_{1}$ & $z_{2}$ & $z_{3}$ & $z_{4}$ & $z_{5}$ & $z_{6}$ & $z_{7}$ & $z_{8}$ & $D_{\mathrm{H}}$ \\
\hline \hline 1 & 0 & 1 & 1 & 1 & 0 & 0 & 4 & 8 \\
\hline 0 & 0 & 0 & 1 & 0 & 1 & 0 & 2 & 4 \\
\hline 0 & 0 & 1 & 0 & 0 & 0 & 1 & 2 & 4 \\
\hline 1 & 1 & 0 & 0 & 0 & 0 & 0 & 2 & 4 \\
\hline
\end{tabular}


and its SR-ideal:

$$
\mathrm{SR}=\left\{z_{6} z_{7}, z_{2} z_{6}, z_{1} z_{2}, z_{4} z_{5}, z_{4} z_{6}, z_{3} z_{7} z_{8}, z_{1} z_{3} z_{5} z_{8}\right\}
$$

The three blow-up divisors are given by $D_{2}, D_{6}$ and $D_{7}$.

The intersection structure on the Calabi-Yau hypersurface in terms of the basis divisors $D_{i}=\left\{z_{i}=0\right\}, i=1, \ldots, 4$ is:

$$
I_{3}=-2 D_{1}^{3}-2 D_{3}^{3}-2 D_{2}^{2} D_{3}+2 D_{3}^{2} D_{2}+2 D_{1} D_{3} D_{4}+2 D_{2} D_{3} D_{4},
$$

while the generators of the Mori cone of the toric four-fold given by (A.1) and (A.2), turn out to be:

$$
\int_{\mathcal{C}_{j}} D_{i}=\left(\begin{array}{cccc}
1 & 0 & 1 & 0 \\
0 & -1 & 0 & 1 \\
-1 & 0 & 0 & 0 \\
1 & 1 & -1 & 0
\end{array}\right) .
$$

We shall now expand the Kähler form in terms of the generators $\Gamma_{i}$ of the Kähler cone as:

$$
J=\sum_{i=1}^{4} r_{i} \Gamma_{i} \quad \text { with } \quad r_{i}>0,
$$

where the generators dual to (A.4), look like:

$$
\Gamma_{1}=2 D_{2}+D_{3}+2 D_{4}-D_{1}, \quad \Gamma_{2}=D_{4}, \quad \Gamma_{3}=D_{2}+D_{4}, \quad \Gamma_{4}=D_{2}+D_{3}+D_{4} .
$$

The volumes of the basis divisors are given by:

$$
\begin{array}{ll}
\tau_{1}=3 r_{1}^{2}+2 r_{4}\left(r_{2}+r_{3}+r_{4}\right)+2 r_{1}\left(r_{2}+r_{3}+3 r_{4}\right), & \tau_{2}=\left(r_{1}+r_{4}\right)\left(r_{1}+2 r_{2}+r_{4}\right), \\
\tau_{3}=\left(r_{1}+r_{3}\right)\left(3 r_{1}+2 r_{2}+r_{3}\right)+2 r_{4}\left(3 r_{1}+r_{2}+2 r_{3}\right)+2 r_{4}^{2}, & \tau_{4}=2\left(r_{1}+r_{4}\right)\left(r_{1}+r_{3}+r_{4}\right),
\end{array}
$$

while the volume of the Calabi-Yau manifold reads:

$$
\begin{aligned}
\mathcal{V}= & r_{1}\left(r_{1}+r_{3}\right)\left[2\left(r_{1}+r_{2}\right)+r_{3}\right]+\left(6 r_{1}^{2}+4 r_{1} r_{2}+6 r_{1} r_{3}+2 r_{2} r_{3}+r_{3}^{2}\right) r_{4} \\
& +\left(6 r_{1}+2 r_{2}+3 r_{3}\right) r_{4}^{2}+\frac{5}{3} r_{4}^{3} .
\end{aligned}
$$

Following the algorithm presented in section 2.2, we find the diagonal basis:

$$
\begin{array}{ll}
D_{a}:=D_{4}, & D_{b}:=2 D_{2}+D_{4}, \\
D_{c}:=2\left(D_{2}+D_{3}-D_{1}\right)+D_{4}, & D_{d}:=D_{2}+D_{4}-D_{1}=D_{6},
\end{array}
$$

where the intersection polynomial reduces to:

$$
I_{3}=8 D_{a} D_{b} D_{c}+2 D_{d}^{3} .
$$

The Calabi-Yau volume simplifies to:

$$
\mathcal{V}=t_{a} \tau_{a}-\beta \tau_{d}^{3 / 2}=\alpha \sqrt{\tau_{a} \tau_{b} \tau_{c}}-\beta \tau_{d}^{3 / 2},
$$

with $\alpha=1 /(2 \sqrt{2})$ and $\beta=1 / 3$. As we shall see in the next section, $\tau_{a}=\tau_{4}$ gives the volume of the $\mathrm{K} 3$ fibre while $\tau_{d}=\tau_{6}$ is the volume of a $\mathrm{dP}_{7}$ diagonal divisor. 


\section{A.1.1 Divisor analysis}

Let us now study the topology of the divisors of the Calabi-Yau three-fold under consideration. The rigid divisors are $D_{2}, D_{5}, D_{6}$ and $D_{7}$. However only $D_{6}$ is a del Pezzo since its topological numbers, $\chi_{h}=1$ and $\chi=10$, indicate that this divisor is a $\mathrm{dP}_{7}$. In order to check this, we project the relevant part of the fan along $z_{6}$ obtaining:

$$
D_{6}: \begin{array}{|c|c|c|c||c|}
\hline z_{5} & z_{1} & z_{3} & z_{8} & \left.D_{\mathrm{H}}\right|_{D_{6}} \\
\hline 1 & 1 & 1 & 2 & 4 \\
\hline
\end{array} \quad \text { with }\left.\quad \mathrm{SR}\right|_{D_{6}}=\left\{z_{1} z_{3} z_{5} z_{8}\right\},
$$

which shows explicitly that $D_{6}$ is a del Pezzo seven surface.

In order to find the K3 fibration we look for the reflexive sections of the N-lattice polytope encoded in (A.1) which admits three sections of this kind even if only one is compatible with the projection. The vectors corresponding to the homogeneous coordinates $z_{4}, z_{5}$ and $z_{6}$ do not lie on this plane. The projection along the plane maps $z_{4}$ to $\zeta_{1}$ and $z_{5} z_{6}$ to $\zeta_{2}$, where $\zeta_{1}$ and $\zeta_{2}$ are the homogeneous coordinates of the $\mathbb{P}^{1}$. This projection does indeed define a toric homomorphism because $\mathrm{SR}_{\mathbb{P} 1}=\left\{\zeta_{1} \zeta_{2}\right\} \simeq\left\{z_{4} z_{5}, z_{4} z_{6}\right\}$ is a subset of (A.2). Over the point $\zeta_{1}=z_{4}=0$, we get a generic K3 fibre and at $\zeta_{2}=0$ the fibre becomes the sum of $D_{5}$ and $D_{6}$. We finally stress that, for this fibration, it is not possible to find a section that embeds the base into the Calabi-Yau three-fold.

Let us summarise the results of our divisor analysis:

\begin{tabular}{|c|c|c|}
\hline divisor classes & $D_{4} \simeq D_{5}+D_{6}$ & $D_{6}$ \\
\hline variety & $\mathrm{K} 3$ & $\mathrm{dP}_{7}$ \\
\hline volume & $2\left(r_{1}+r_{4}\right)\left(r_{1}+r_{3}+r_{4}\right)$ & $r_{4}^{2}$ \\
\hline
\end{tabular}

\section{A.1.2 Orientifolding}

Let us now briefly discuss the case of the Calabi-Yau orientifold with $z_{8} \leftrightarrow-z_{8}$ involution. The fixed point set of the ambient space is given by:

$$
\left.\{\text { Fixed }\}\right|_{z_{8} \leftrightarrow-z_{8}}=\left\{D_{8}, D_{3} \cdot D_{7}, D_{1} \cdot D_{3} \cdot D_{5} \cdot D_{6}\right\} \text {. }
$$

However, only $D_{8}$ intersects the invariant hypersurface, and so we get only an $O 7$-plane.

Regarding the Calabi-Yau hypersurface, also the K3 fibre and the del Pezzo seven surface become less generic in the orientifold case even if, together with all the other toric divisors, they do not degenerate.

For the F-theory uplift, the stringy Euler number of the four-fold is 14688 which is in agreement with a single Whitney brane configuration.

\section{A.2 Another example with one del Pezzo divisor}

The second example that we work out in detail in this appendix, is a K3 fibration which admits only one toric del Pezzo divisor. The fan of the toric four-fold is defined in terms 
of its weight matrix:

\begin{tabular}{|c|c|c|c|c|c|c|c||c|}
\hline$z_{1}$ & $z_{2}$ & $z_{3}$ & $z_{4}$ & $z_{5}$ & $z_{6}$ & $z_{7}$ & $z_{8}$ & $D_{\mathrm{H}}$ \\
\hline \hline 0 & 1 & 0 & 0 & 2 & 2 & 6 & 1 & 12 \\
\hline 0 & 0 & 0 & 1 & 1 & 1 & 3 & 0 & 6 \\
\hline 0 & 0 & 1 & 0 & 0 & 1 & 2 & 0 & 4 \\
\hline 1 & 1 & 0 & 0 & 1 & 0 & 3 & 0 & 6 \\
\hline
\end{tabular}

and its SR-ideal:

$$
\mathrm{SR}=\left\{z_{1} z_{2}, z_{2} z_{8}, z_{1} z_{3}, z_{1} z_{4}, z_{4} z_{5}, z_{3} z_{6} z_{7}, z_{5} z_{6} z_{7} z_{8}\right\}
$$

With these data we can calculate the intersection structure on the Calabi-Yau hypersurface in terms of the basis divisors $D_{i}=\left\{z_{i}=0\right\}, i=1, \ldots, 4$ :

$$
I_{3}=D_{1}^{3}-2 D_{2} D_{3}^{2}+4 D_{3}^{3}+2 D_{2} D_{3} D_{4}-4 D_{3} D_{4}^{2} .
$$

The Mori cone generators of the toric four-fold given by (A.13) and (A.14) are:

$$
\int_{\mathcal{C}_{j}} D_{i}=\left(\begin{array}{cccc}
-1 & 0 & 0 & 0 \\
0 & 1 & 0 & -2 \\
0 & 0 & -3 & 2 \\
2 & 0 & 3 & 0
\end{array}\right):=\mathcal{M}_{j i}
$$

while the dual generators to (6.24) look like:

$$
\Gamma_{1}=6 D_{2}-3 D_{1}+2 D_{3}+3 D_{4}, \quad \Gamma_{2}=D_{2}, \quad \Gamma_{3}=2 D_{2}+D_{4}, \quad \Gamma_{4}=6 D_{2}+2 D_{3}+3 D_{4} .
$$

Expanding the Kähler form in terms of the Г's as:

$$
J=\sum_{i=1}^{4} r_{i} \Gamma_{i}, \quad \text { with } \quad r_{i}>0,
$$

the divisor volumes turn out to be:

$$
\begin{array}{ll}
\tau_{1}=\frac{9}{2} r_{1}^{2}, & \tau_{2}=4\left(r_{1}+r_{4}\right)\left(2 r_{1}+r_{3}+2 r_{4}\right), \\
\tau_{3}=2\left(r_{1}+r_{3}+r_{4}\right)\left(r_{1}+r_{2}+r_{3}+r_{4}\right), & \tau_{4}=4 r_{2}\left(r_{1}+r_{4}\right)
\end{array}
$$

whereas the volume of the Calabi-Yau manifold becomes:

$$
\begin{aligned}
\mathcal{V}= & \frac{77}{6} r_{1}^{3}+4 r_{3}\left(r_{2}+r_{3}\right) r_{4}+8\left(r_{2}+2 r_{3}\right) r_{4}^{2}+\frac{52}{3} r_{4}^{3}+4 r_{1}^{2}\left(2 r_{2}+4 r_{3}+13 r_{4}\right) \\
& +4 r_{1}\left[r_{3}\left(r_{2}+r_{3}\right)+4\left(r_{2}+2 r_{3}\right) r_{4}+13 r_{4}^{2}\right] .
\end{aligned}
$$

These expressions simplify considerably if we expand the Kähler form in terms of the initial basis $D_{i}, i=1, \ldots, 4$ as:

$$
J=\sum_{i=1}^{4} t_{i} D_{i}, \quad \text { with } \quad \sum_{i=1}^{4} \mathcal{M}_{j i} t_{i}>0,
$$


since they become:

$$
\begin{array}{ll}
\tau_{1}=\frac{1}{2} t_{1}^{2}, & \tau_{2}=t_{3}\left(2 t_{4}-t_{3}\right), \\
\tau_{3}=2\left(t_{3}-t_{4}\right)\left(t_{3}+t_{4}-t_{2}\right), & \tau_{4}=2 t_{3}\left(t_{2}-2 t_{4}\right),
\end{array}
$$

and:

$$
\begin{aligned}
\mathcal{V} & =\frac{1}{3} t_{3}\left(2 t_{3}^{2}-3 t_{2} t_{3}+6 t_{2} t_{4}-6 t_{4}^{2}\right)+\frac{1}{6} t_{1}^{3} \\
& =\left(t_{2}-2 t_{4}\right) \tau_{2}+\frac{2}{3} t_{3}\left(t_{3}^{2}-3 t_{3} t_{4}+3 t_{4}^{2}\right)-\frac{\sqrt{2}}{3} \tau_{1}^{3 / 2}
\end{aligned}
$$

Similarly to the previous example, we have not been able to write the volume just in terms of Kähler moduli $\tau_{i}$. Thus we did not manage to diagonalise the volume even if the modulus $\tau_{1}$ is manifestly diagonal. As we shall see in the next section, the divisor $D_{1}$ is a del Pezzo eight surface.

Let us now show that we can indeed write down the volume form just in terms of the $\tau$ 's in the anisotropic large volume limit used in $[28,29]$ for promising applications to particle phenomenology. We start by taking the limit of small $\tau_{1}$. As can be seen from (A.19), this is equivalent to sending $r_{1} \rightarrow 0$. In this limit, the divisor volumes become:

$$
\tau_{1} \rightarrow 0, \quad \tau_{2} \rightarrow 4 r_{4}\left(r_{3}+2 r_{4}\right), \quad \tau_{3} \rightarrow 2\left(r_{3}+r_{4}\right)\left(r_{2}+r_{3}+r_{4}\right), \quad \tau_{4} \rightarrow 4 r_{2} r_{4},
$$

while the Calabi-Yau volume reduces to:

$$
\mathcal{V} \rightarrow r_{2} \tau_{2}+4 r_{3} r_{4}\left(r_{3}+4 r_{4}\right)+\frac{52}{3} r_{4}^{3} .
$$

As we shall see in the next section, the volume of the K3 fibre is given by $\tau_{2}$ while the volume of the $\mathbb{P}^{1}$ base of the fibration is controlled by the Kähler parameter $r_{2}=t_{2}-2 t_{4}$. Therefore the anisotropic limit $r_{2} \gg \sqrt{\tau_{2}}$ corresponds to $r_{2} \gg r_{3} \sim r_{4}$. In this new limit the expressions (A.24) and (A.25) further simplify to:

$$
\tau_{1} \rightarrow 0, \quad \tau_{2} \rightarrow 4 r_{4}\left(r_{3}+2 r_{4}\right), \quad \tau_{3} \rightarrow 2 r_{2}\left(r_{3}+r_{4}\right), \quad \tau_{4} \rightarrow 4 r_{2} r_{4}, \quad \mathcal{V} \rightarrow r_{2} \tau_{2}
$$

We can now invert the expression of the $r$ 's in terms of the $\tau$ 's obtaining:

$$
r_{2}=\frac{\sqrt{\tau_{4}\left(2 \tau_{3}+\tau_{4}\right)}}{2 \sqrt{\tau_{2}}}, \quad r_{3}=\frac{\sqrt{\tau_{2}}\left(2 \tau_{3}-\tau_{4}\right)}{2 \sqrt{\tau_{4}\left(2 \tau_{3}+\tau_{4}\right)}}, \quad r_{4}=\frac{\sqrt{\tau_{2} \tau_{4}}}{2 \sqrt{2 \tau_{3}+\tau_{4}}}
$$

and so the Calabi-Yau volume in the limit $r_{2} \gg r_{3} \sim r_{4} \gg r_{1}>0$ takes the final form:

$$
\mathcal{V}=\frac{1}{2} \sqrt{\tau_{2} \tau_{4}\left(2 \tau_{3}+\tau_{4}\right)}-\frac{\sqrt{2}}{3} \tau_{1}^{3 / 2}
$$

\section{A.2.1 Divisor analysis}

In this section we shall analyse the topology of the divisors of the Calabi-Yau three-fold under consideration. The rigid divisors are $D_{1}, D_{3}, D_{4}$ and $D_{8}$. However only $D_{1}$ is a del 
Pezzo since its topological numbers, $\chi_{h}=1$ and $\chi=11$, indicate that this divisor is a $\mathrm{dP}_{8}$. In order to check this, we project the relevant part of the fan along $z_{1}$ obtaining:

$$
D_{1}: \begin{array}{|c|c|c|c||c|}
\hline z_{5} & z_{6} & z_{7} & z_{8} & \left.D_{\mathrm{H}}\right|_{D_{1}} \\
\hline 1 & 2 & 3 & 1 & 6 \\
\hline
\end{array} \quad \text { with }\left.\quad \mathrm{SR}\right|_{D_{1}}=\left\{z_{5} z_{6} z_{7} z_{8}\right\},
$$

which shows explicitly that $D_{1}$ is a del Pezzo eight surface.

In order to find the K3 fibration we look for the reflexive sections of the N-lattice polytope encoded in (A.13) which admits two sections of this kind even if only one is compatible with the projection. The vectors corresponding to the homogeneous coordinates $z_{1}, z_{2}$ and $z_{8}$ do not lie on this plane. The projection along the plane maps $z_{2}$ to $\zeta_{1}$ and $z_{1} z_{8}$ to $\zeta_{2}$, where $\zeta_{1}$ and $\zeta_{2}$ are the homogeneous coordinates of the $\mathbb{P}^{1}$. This projection does indeed define a toric homomorphism because $\operatorname{SR}_{\mathbb{P}^{1}}=\left\{\zeta_{1} \zeta_{2}\right\} \simeq\left\{z_{1} z_{2}, z_{2} z_{8}\right\}$ is a subset of (A.14). Over the point $\zeta_{1}=z_{2}=0$, we get a generic K3 fibre and at $\zeta_{2}=0$ the fibre becomes the sum of $D_{1}$ and $D_{8}$.

We finally stress that for this fibration we also have global sections of the base. Given that the two $\mathbb{P}^{1}$ 's turn out to be $\mathcal{C}_{2 \mathbb{P}^{1}}=D_{4} \cap D_{6}$, the volume of the base is:

$$
\operatorname{Vol}(\text { base })=\frac{1}{2} \operatorname{Vol}\left(\mathcal{C}_{2 \mathbb{P}^{1}}\right)=\frac{1}{2} \int_{D_{3} \cap D_{6}} \imath^{*} J=\frac{1}{2} D_{4} \cdot D_{6} \cdot J=r_{2}=t_{2}-2 t_{4} .
$$

Let us summarise the results of our divisor analysis:

\begin{tabular}{|c|c|c|}
\hline divisor classes & $D_{1}$ & $D_{2} \simeq D_{1}+D_{8}$ \\
\hline variety & $\mathrm{dP}_{8}$ & $\mathrm{~K} 3$ \\
\hline volume & $\frac{9}{2} r_{1}^{2}$ & $4\left(r_{1}+r_{4}\right)\left(2 r_{1}+r_{3}+2 r_{4}\right)$ \\
\hline
\end{tabular}

\section{A.2.2 Orientifolding}

Let us now briefly discuss the orientifold with $z_{7} \leftrightarrow-z_{7}$ involution. The fixed point set of the ambient space is given by:

$$
\left.\{\text { Fixed }\}\right|_{z_{7} \leftrightarrow-z_{7}}=\left\{D_{7}, D_{1} \cdot D_{5} \cdot D_{8}, D_{1} \cdot D_{5} \cdot D_{6} \cdot D_{8}\right\} .
$$

However, only $D_{7}$ and $D_{1} \cdot D_{5} \cdot D_{8}$ intersect the invariant hypersurface, and so we get an $O 7$-plane and an $O 3$-plane.

Regarding the Calabi-Yau hypersurface, also the K3 fibre and the del Pezzo eight surface become less generic in the orientifold case even if, together with all the other toric divisors, they do not degenerate.

The stringy Euler characteristic of the geometry of the F-theory uplift is 14136. This matches the $D 3$-tadpole of a single Whitney brane configuration including the $O 3$-plane.

\section{B List of all the K3 fibrations with diagonal del Pezzo divisors}

In this appendix we give the full list of the 158 polytopes that admit a K3-fibred Calabi-Yau three-fold with $h^{1,1}=4$ Kähler moduli and at least one 'diagonal' del Pezzo divisor. 
The lattice polytopes are described in terms of their weight matrices. All their topological data can be obtained via the $\mathrm{C}$ program mori.x [61]. For example, the command to obtain the information about the Calabi-Yau three-fold coming from the triangulation of the polytope number 94 is:

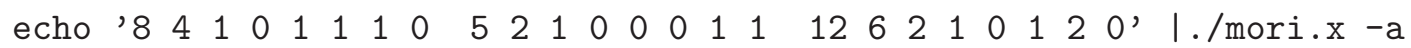

Notice that the example of section 5.1 corresponds to the polytope number 1 , the example discussed in section 6.1 is the 94 th polytope, the example of section 6.2 corresponds to the polytope number 136, the example described in section A.1 is given by the 147th polytope, while the example of section A.2 corresponds to the polytope number 79 . We stress that the difference between the weight matrices of the polytopes listed here and those defining the toric ambient varieties of the examples presented in the main text, is due to the fact that we are dealing with reflexive lattice polytopes which can be defined without having to specify all the weights.

\begin{tabular}{|c|c|c|c|}
\hline$\#$ & $\Sigma_{i} w_{i}$ & $w_{i}$ & $\mathbb{Z}_{2}$ action \\
\hline 1 & 8 & $\begin{array}{lllll}2 & 2 & 2 & 1 & 1\end{array}$ & 0010110 \\
\hline 2 & 8 & 22211 & 11110 \\
\hline 3 & 12 & 22611 & 10010 \\
\hline 4 & 12 & 22611 & 11110 \\
\hline 5 & 12 & 22611 & 000110 \\
\hline
\end{tabular}

\begin{tabular}{|c|c|c|c|c|}
\hline \# & $\overline{\Sigma_{i} w_{i}^{A}}$ & $w_{i}^{A}$ & $\Sigma_{i} w_{i}^{B}$ & $w_{i}^{B}$ \\
\hline 6 & 8 & 2221120 & 2 & $\begin{array}{lllllll} & 0 & 0 & 1 & 0 & 0 & 1\end{array}$ \\
\hline 7 & 6 & 131001 & 4 & $\begin{array}{lllll}0 & 1 & 0 & 1 & 2\end{array}$ \\
\hline 8 & 8 & 131102 & 6 & 130110 \\
\hline 9 & 6 & 212001 & 6 & 31010110 \\
\hline 10 & 6 & 121101 & 12 & 341022 \\
\hline 11 & 8 & 411011 & 18 & 930132 \\
\hline 12 & 4 & 201001 & 12 & 621120 \\
\hline 13 & 5 & 111011 & 12 & 332130 \\
\hline 14 & 6 & 111012 & 12 & 313230 \\
\hline 15 & 6 & 103110 & 12 & 215202 \\
\hline 16 & 12 & 333102 & 12 & 330231 \\
\hline 17 & 14 & 173012 & 16 & 184120 \\
\hline 18 & 10 & 510211 & 18 & 932013 \\
\hline 19 & 12 & 620211 & 14 & 721202 \\
\hline 20 & 18 & 393102 & 18 & 390231 \\
\hline 21 & 18 & 6991011 & 24 & 8120121 \\
\hline
\end{tabular}




\begin{tabular}{|c|c|c|c|c|c|}
\hline \# & $\overline{\Sigma_{i} w_{i}^{A}}$ & $\overline{w_{i}^{A}}$ & $\overline{\Sigma_{i} w_{i}^{B}}$ & $w_{i}^{B}$ & $\mathbb{Z}_{2}$ action on $\mathrm{B}$ \\
\hline 22 & 4 & $\begin{array}{llllll}1 & 1 & 0 & 0 & 1\end{array}$ & 2 & $\begin{array}{lllllll} & 0 & 0 & 1 & 1 & 0\end{array}$ & 111100 \\
\hline 23 & 4 & 111001 & 4 & 110110 & 0011100 \\
\hline 24 & 4 & $\begin{array}{llllll}1 & 1 & 1 & 0 & 0 & 1\end{array}$ & 4 & $\begin{array}{lllllll}1 & 0 & 1 & 1\end{array}$ & $\begin{array}{lllllll}1 & 0 & 0 & 1 & 0 & 0\end{array}$ \\
\hline 25 & 6 & 131010 & 4 & $\begin{array}{llllll}0 & 2 & 0 & 1 & 0 & 1\end{array}$ & 010100 \\
\hline 26 & 6 & 130110 & 4 & $\begin{array}{lllll}0 & 2 & 1 & 0 & 0\end{array}$ & $\begin{array}{llllllllll}0 & 0 & 1 & 1 & 0 & 0\end{array}$ \\
\hline 27 & 6 & 131001 & 6 & 130110 & 001100 \\
\hline 28 & 6 & 131001 & 6 & 130110 & 100100 \\
\hline 29 & 8 & $\begin{array}{llllll}4 & 1 & 0 & 1 & 1 & 1\end{array}$ & 6 & $\begin{array}{llllll}3 & 1 & 1 & 0 & 0 & 1\end{array}$ & 111100 \\
\hline 30 & 8 & 411101 & 6 & $\begin{array}{llllll}3 & 1 & 0 & 1 & 1 & 0\end{array}$ & 101000 \\
\hline
\end{tabular}

\begin{tabular}{|c|c|c|c|c|c|c|}
\hline$\#$ & $\sum_{i} w_{i}^{A}$ & $w_{i}^{A}$ & $\sum_{i} w_{i}^{B}$ & $w_{i}^{B}$ & $\Sigma_{i} w_{i}^{C}$ & $w_{i}^{C}$ \\
\hline 31 & 12 & 1162002 & 6 & $\begin{array}{lllllll}1 & 0 & 3 & 0 & 1 & 0 & 1\end{array}$ & 6 & 010131010 \\
\hline 32 & 10 & 1510102 & 6 & $\begin{array}{lllllll}0 & 3 & 0 & 1 & 1 & 0 & 1\end{array}$ & 8 & 2410010 \\
\hline 33 & 5 & $\begin{array}{llllll}1 & 1 & 0 & 1 & 0 & 1\end{array}$ & 3 & 011100010 & 8 & 1221200 \\
\hline 34 & 6 & 2111010 & 3 & 1010001 & 6 & 2200110 \\
\hline 35 & 6 & 1210011 & 6 & 210101011 & 6 & $\begin{array}{lllllll}2 & 0 & 0 & 2 & 1 & 0 & 1\end{array}$ \\
\hline 36 & 8 & 2112002 & 4 & 1100101 & 4 & 1011010 \\
\hline 37 & 8 & 1220210 & 4 & 111100001 & 4 & 1002010 \\
\hline 38 & 5 & 1110101 & 4 & 1101010 & 6 & 1110012 \\
\hline 39 & 6 & 1102110 & 3 & 000011101 & 4 & 1110010 \\
\hline 40 & 7 & 21102201 & 6 & 2101200 & 8 & 2121020 \\
\hline 41 & 10 & 1510012 & 4 & 0210100 & 8 & 2401010 \\
\hline 42 & 5 & $\begin{array}{lllllll}1 & 1 & 0 & 0 & 1 & 1\end{array}$ & 2 & $\begin{array}{lllllll}0 & 0 & 0 & 0 & 1 & 0 & 1\end{array}$ & 5 & 1101110 \\
\hline 43 & 8 & 2210012 & 2 & 00000101 & 4 & 1101010 \\
\hline 44 & 5 & 1110101 & 4 & 1110010 & 8 & 2121200 \\
\hline 45 & 6 & 1101210 & 2 & 00000101 & 4 & 1110010 \\
\hline 46 & 7 & 1311010 & 6 & 1301100 & 7 & 1310101 \\
\hline 47 & 10 & 1510102 & 6 & 1310010 & 6 & 0301101 \\
\hline 48 & 8 & 1410101 & 6 & 1301010 & 10 & 1510012 \\
\hline 49 & 6 & 1210101 & 2 & 01000010 & 10 & 1421200 \\
\hline 50 & 8 & 1411001 & 10 & 1520011 & 12 & 2612100 \\
\hline 51 & 8 & 1301120 & 6 & 1310100 & 10 & 1501102 \\
\hline 52 & 6 & 1210020 & 6 & 1301100 & 8 & 1410002 \\
\hline 53 & 8 & 1401101 & 6 & 1310010 & 14 & 2720102 \\
\hline 54 & 12 & 6120102 & 6 & $\begin{array}{lllllll}3 & 1 & 0 & 0 & 0 & 1\end{array}$ & 8 & 4100120 \\
\hline 55 & 4 & 1110001 & 4 & 1101010 & 4 & 2000110 \\
\hline 56 & 7 & 2210110 & 3 & 11000001 & 8 & 2201210 \\
\hline 57 & 14 & 7310012 & 8 & 4210100 & 8 & 4201010 \\
\hline 58 & 8 & 4101101 & 8 & 4210010 & 10 & 5110102 \\
\hline 59 & 7 & 2210011 & 4 & 1110100 & 6 & 2201010 \\
\hline 60 & 8 & $\begin{array}{lllllll}4 & 1 & 1 & 0 & 1 & 0 & 1\end{array}$ & 8 & 4101200 & 10 & 5120110 \\
\hline
\end{tabular}




\begin{tabular}{|c|c|c|c|c|c|c|}
\hline \# & $\sum_{i} w_{i}^{A}$ & $w_{i}^{A}$ & $\Sigma_{i} w_{i}^{B}$ & $w_{i}^{B}$ & $\Sigma_{i} w_{i}^{C}$ & $w_{i}^{C}$ \\
\hline 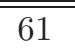 & 10 & 2501101 & 8 & 22410010 & 14 & 23710102 \\
\hline 62 & 8 & 1411010 & 8 & 2400110 & 10 & 0521011 \\
\hline 63 & 10 & 5101210 & 4 & $\begin{array}{llllllll}2 & 0 & 0 & 0 & 1 & 0 & 1\end{array}$ & 8 & 4210010 \\
\hline 64 & 6 & 1201110 & 5 & 1210010 & 6 & $\begin{array}{llllll}13 & 10 & 0 & 0 & 1\end{array}$ \\
\hline 65 & 5 & $\begin{array}{lllllll}2 & 1 & 0 & 1 & 0 & 0 & 1\end{array}$ & 4 & 20000110 & 5 & $\begin{array}{lllllllllll}2 & 0 & 1 & 0 & 1 & 0 & 1\end{array}$ \\
\hline 66 & 10 & 25011020 & 4 & $\begin{array}{lllllll}0 & 2 & 1 & 0 & 0 & 0 & 1\end{array}$ & 10 & 0501202 \\
\hline 67 & 7 & $\begin{array}{lllllll}30 & 1 & 1 & 1 & 1\end{array}$ & 4 & $\begin{array}{lllllll}2 & 1 & 0 & 0 & 0 & 0 & 1\end{array}$ & 5 & $\begin{array}{llllllll}2 & 0 & 0 & 1 & 1 & 0 & 1\end{array}$ \\
\hline 67 & 5 & 1201100 & 3 & 01110010 & 6 & $\begin{array}{lllll}13 & 0 & 0 & 1001\end{array}$ \\
\hline 67 & 8 & 4110111110 & 6 & $\begin{array}{lllllll}3 & 1 & 1 & 0 & 1 & 0\end{array}$ & 6 & $\begin{array}{llllllll}3 & 0 & 1 & 0 & 1 & 0 & 1\end{array}$ \\
\hline 70 & 14 & 7202210 & 6 & $\begin{array}{llllllll}3 & 1 & 0 & 0 & 1 & 0 & 1\end{array}$ & 6 & $\begin{array}{llllll}30 & 2 & 0 & 0 & 1 & 0\end{array}$ \\
\hline 71 & 6 & 1121100 & 6 & 1130010 & 8 & 1140101 \\
\hline 72 & 6 & 1121010 & 6 & 1031100 & 7 & 1031011 \\
\hline 73 & 14 & 2710022 & 6 & 1301010 & 10 & 2510200 \\
\hline 74 & 12 & 2252010 & 6 & 1031100 & 14 & 2072012 \\
\hline 75 & 6 & 1310100 & 4 & $\begin{array}{lllllllll}0 & 1 & 0 & 1 & 0 & 2 & 0\end{array}$ & 6 & $\begin{array}{llllll}0 & 30 & 1 & 0 & 0\end{array}$ \\
\hline 76 & 7 & 311010101 & 3 & $\begin{array}{llllll}10 & 10 & 0 & 10\end{array}$ & 6 & 3101100 \\
\hline 77 & 6 & 1310100 & 2 & $\begin{array}{llllllll}0 & 0 & 0 & 1 & 0 & 1 & 0\end{array}$ & 4 & $\begin{array}{lllllll}0 & 2 & 0 & 1 & 0 & 0 & 1\end{array}$ \\
\hline 78 & 6 & 1310100 & 2 & $\begin{array}{lllllllll}0 & 0 & 1 & 0 & 1 & 0\end{array}$ & 3 & $\begin{array}{llllllllll}0 & 1 & 0 & 0 & 0 & 1 & 1\end{array}$ \\
\hline 79 & 12 & 6210120 & 4 & $\begin{array}{lllllll}2 & 1 & 0 & 0 & 0 & 0 & 1\end{array}$ & 6 & 3011010 \\
\hline 80 & 8 & 411100011 & 4 & $\begin{array}{llllllll}2 & 0 & 0 & 0 & 1 & 0 & 1\end{array}$ & 8 & 4101110 \\
\hline 81 & 5 & 1210010 & 5 & 1201100 & 6 & $\begin{array}{llllll}13 & 0 & 0 & 1 & 0 & 1\end{array}$ \\
\hline 82 & 6 & $\begin{array}{llllllll}3 & 0 & 1 & 1 & 1 & 0\end{array}$ & 3 & $\begin{array}{lllllll}1 & 1 & 0 & 0 & 0 & 0 & 1\end{array}$ & 4 & $\begin{array}{llllllll}2 & 0 & 1 & 1 & 0 & 0\end{array}$ \\
\hline 83 & 7 & 1310110 & 5 & 12011100 & 6 & $\begin{array}{lllllll}1 & 3 & 0 & 0 & 1 & 0 & 1\end{array}$ \\
\hline 84 & 8 & 411100011 & 6 & $\begin{array}{lllllll}3 & 1 & 0 & 1 & 0 & 1 & 0\end{array}$ & 6 & $\begin{array}{llll}30 & 20 & 1 & 0\end{array}$ \\
\hline 85 & 7 & 1301110 & 8 & 1401101 & 12 & 26112000 \\
\hline 86 & 10 & 5201011 & 8 & 4210010 & 14 & 7302110 \\
\hline 87 & 10 & 250011110 & 6 & 1300101 & 10 & 255100020 \\
\hline 88 & 14 & 2720021 & 6 & 1310100 & 6 & 1301010 \\
\hline 89 & 8 & 41110011 & 6 & $\begin{array}{llllll}3 & 1 & 0 & 1 & 0 & 1\end{array}$ & 8 & 4210100 \\
\hline 90 & 10 & 5201110 & 4 & 2000101 & 8 & 4210010 \\
\hline 91 & 14 & 7301210 & 6 & $\begin{array}{lllllll}3 & 1 & 0 & 0 & 1 & 0 & 1\end{array}$ & 8 & 4210010 \\
\hline 92 & 7 & $\begin{array}{llllll}3 & 1 & 1 & 0 & 1 & 0\end{array}$ & 4 & $\begin{array}{llllllllll}2 & 0 & 1 & 1 & 0 & 0\end{array}$ & 6 & $\begin{array}{lllllll}3 & 0 & 0 & 1 & 1 & 0\end{array}$ \\
\hline 93 & 8 & 1400111 & 10 & 25100020 & 12 & $26 \begin{array}{lllll}2 & 1 & 1 & 2 & 0\end{array}$ \\
\hline 94 & 8 & 4101110 & 5 & 21000011 & 12 & 6210120 \\
\hline 95 & 8 & 1410101 & 4 & 02100010 & 12 & 1621200 \\
\hline 96 & 5 & $\begin{array}{lllllllll}2 & 0 & 1 & 1 & 0 & 1\end{array}$ & 4 & $\begin{array}{lllllll}2 & 1 & 0 & 0 & 0 & 0 & 1\end{array}$ & 6 & $\begin{array}{lllllll}3 & 0 & 1 & 0 & 0 & 1 & 1\end{array}$ \\
\hline 97 & 11 & 2510210 & 5 & 1201100 & 6 & $\begin{array}{llllll}13 & 0 & 0 & 1 & 0 & 1\end{array}$ \\
\hline 98 & 11 & 2521100 & 5 & 1210010 & 12 & $\begin{array}{llllll}2 & 62 & 1 & 0 & 0 & 1\end{array}$ \\
\hline 99 & 10 & 5210110 & 4 & $\begin{array}{lllllll}2 & 1 & 0 & 0 & 0 & 0 & 1\end{array}$ & 12 & 6201210 \\
\hline 100 & 10 & 25510011 & 6 & 1310100 & 8 & 2401010 \\
\hline 101 & 12 & $2 \begin{array}{lllll}2 & 0 & 0 & 1 & 2\end{array}$ & 10 & 2510020 & 16 & 3801130 \\
\hline 102 & 18 & $\begin{array}{llllllll}6 & 9 & 0 & 1 & 1 & 1\end{array}$ & 12 & 4610010 & 12 & $\begin{array}{llllllll}4 & 6 & 0 & 0 & 1 & 0 & 1\end{array}$ \\
\hline 103 & 8 & 4110101 & 6 & 3110010 & 12 & 6211200 \\
\hline 104 & 6 & $\begin{array}{llllll}31 & 0 & 0 & 10 & 1\end{array}$ & 6 & 3011001 & 8 & 40020011 \\
\hline
\end{tabular}




\begin{tabular}{|c|c|c|c|c|c|c|}
\hline$\#$ & $\sum_{i} w_{i}^{A}$ & $w_{i}^{A}$ & $\sum_{i} w_{i}^{B}$ & $w_{i}^{B}$ & $\sum_{i} w_{i}^{C}$ & $w_{i}^{C}$ \\
\hline 105 & 10 & $\begin{array}{lllllll}5 & 1 & 0 & 1 & 2 & 1 & 0\end{array}$ & 4 & $\begin{array}{llllllll}2 & 0 & 0 & 0 & 1 & 0 & 1\end{array}$ & 6 & 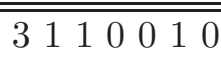 \\
\hline 106 & 8 & 1141010 & 4 & $\begin{array}{lllllll}0 & 1 & 2 & 0 & 0 & 0 & 1\end{array}$ & 8 & 2040110 \\
\hline 107 & 8 & 1401110 & 4 & 0200101 & 10 & 2510020 \\
\hline
\end{tabular}

\begin{tabular}{|c|c|c|c|c|c|c|c|c|}
\hline$\#$ & $\Sigma_{A}$ & $w_{i}^{A}$ & $\Sigma_{B}$ & $w_{i}^{B}$ & $\Sigma_{C}$ & $w_{i}^{C}$ & $\Sigma_{D}$ & $w_{i}^{D}$ \\
\hline 108 & 4 & $\begin{array}{llllllll}1 & 0 & 0 & 0 & 1 & 0 & 1\end{array}$ & 4 & $\begin{array}{llllllll}0 & 0 & 1 & 1 & 0 & 0 & 1\end{array}$ & 4 & $\begin{array}{lllllllll}1 & 0 & 0 & 1 & 0 & 1 & 1 & 0\end{array}$ & 4 & $\begin{array}{lllllllll}0 & 1 & 1 & 0 & 1 & 0 & 0\end{array}$ \\
\hline & & & & & & & & \\
\hline & 0 & & & & & & & \\
\hline & 3 & & 3 & & 3 & & & \\
\hline & 5 & & & & 3 & & & \\
\hline & 5 & & 2 & & 3 & & & \\
\hline & 5 & & & & & & & \\
\hline & 4 & 10 & 2 & & & & & \\
\hline & 5 & & & & 4 & & 4 & \\
\hline & 5 & & 2 & & : & & & \\
\hline & 4 & & 3 & 0 & 3 & & 4 & \\
\hline & 5 & & 2 & & 4 & & 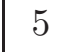 & \\
\hline 20 & 5 & & 4 & 0 & 4 & & 4 & 00 \\
\hline & 6 & & 2 & & 2 & & 2 & \\
\hline ? & 6 & 0 & 3 & 1 & 3 & & : & \\
\hline & 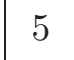 & & 3 & & 4 & & 4 & \\
\hline 24 & 0 & & 4 & & 4 & & c & \\
\hline & 7 & 100 & 3 & & 4 & & 4 & \\
\hline & 6 & & 2 & & 2 & & & \\
\hline & 8 & & 6 & & 6 & & 6 & \\
\hline & 6 & & 2 & & 5 & & & \\
\hline & 7 & & 6 & & 7 & & 8 & \\
\hline & 10 & 100 & 4 & 8 & 4 & 02011000 & 4 & $\begin{array}{llll}0 & 1 & 1 & 1\end{array}$ \\
\hline & 7 & 100 & 2 & & 3 & & & 101 \\
\hline & 8 & 110 & 4 & 1 & 4 & 2001100 & 10 & 5110 \\
\hline & 10 & 210 & 4 & & 4 & 20001110 & 6 & $\begin{array}{llll}0 & 1 & 0 & 1\end{array}$ \\
\hline & 6 & 12110100 & 5 & 12011000 & 6 & 13300101001 & 7 & 0110 \\
\hline & 5 & & 4 & & 5 & & c & 01 \\
\hline & 8 & & 4 & & 6 & & 6 & $\begin{array}{lllllll}0 & 1 & 0 & 0 & 0 & 1\end{array}$ \\
\hline & 5 & & 3 & & 4 & & 6 & 13001010 \\
\hline & 6 & & 6 & & 7 & & 7 & 1301101 \\
\hline & 8 & 04110110 & 4 & 12000001 & 6 & 13010010 & 6 & 0301110 \\
\hline
\end{tabular}




\begin{tabular}{|c|c|c|c|c|c|c|c|c|}
\hline$\#$ & $\Sigma_{A}$ & $w_{i}^{A}$ & $\Sigma_{B}$ & $w_{i}^{B}$ & $\Sigma_{C}$ & $w_{i}^{C}$ & $\Sigma_{D}$ & $w_{i}^{D}$ \\
\hline 140 & 8 & 14101001 & 4 & $\begin{array}{lllllllll} & 2 & 0 & 0 & 0 & 1 & 1\end{array}$ & 6 & 133100100 & 6 & \begin{tabular}{|llllllll}
1 & 3 & 0 & 1 & 1 & 0 & 0 & 0
\end{tabular} \\
\hline 141 & 6 & 0001010 & 4 & $\begin{array}{lllllllll}2 & 0 & 1 & 0 & 0 & 0 & 0 & 1\end{array}$ & 6 & 01110010 & 6 & $\begin{array}{llllllll}3 & 0 & 1 & 0 & 1 & 1 & 0 & 0\end{array}$ \\
\hline 142 & 7 & 30111100 & 3 & $\begin{array}{lllllllllll}0 & 1 & 1 & 0 & 0 & 1 & 0 & 0\end{array}$ & 4 & $\begin{array}{llllllll}0 & 2 & 1 & 0 & 0 & 0 & 0 & 1\end{array}$ & 6 & 01010 \\
\hline 143 & 7 & 31011100 & 5 & 21001010 & 6 & 311000100 & 6 & $\begin{array}{llllll}3 & 10 & 0 & 0 & 0 & 1\end{array}$ \\
\hline 144 & 6 & 1331010000 & 2 & $\begin{array}{llllllll}0 & 0 & 0 & 1 & 0 & 1 & 0 & 0\end{array}$ & 3 & $\begin{array}{llllllll}0 & 1 & 0 & 1 & 0 & 0 & 0 & 1\end{array}$ & 3 & $\begin{array}{llllllll}0 & 1 & 0 & 0 & 0 & 1 & 10\end{array}$ \\
\hline 145 & 8 & $\begin{array}{llllllll}4 & 1 & 1 & 0 & 1 & 0 & 0 & 1\end{array}$ & 4 & $\begin{array}{lllllll}2 & 0 & 1 & 0 & 0 & 0 & 1\end{array}$ & 4 & 200000 & 6 & 01110 \\
\hline 146 & 5 & 12100100 & 5 & 12011000 & 6 & 133100001 & 6 & 130001010 \\
\hline 147 & 8 & 14001110 & 4 & $\begin{array}{lllllll}1 & 2 & 0 & 0 & 0 & 0 & 0\end{array}$ & 4 & $\begin{array}{llllll}0 & 2 & 1 & 0 & 0 & 0\end{array}$ & 4 & 00 \\
\hline 148 & 8 & 14100110 & 4 & 0210101000 & 5 & 12010100 & 6 & $\begin{array}{llllllll}1 & 3 & 0 & 0 & 0 & 1 & 0 & 1\end{array}$ \\
\hline 149 & 6 & 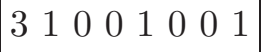 & 4 & 210000010 & 4 & 2010010100 & 6 & $\begin{array}{llllll}0 & 1 & 1 & 1 & 0 & 0\end{array}$ \\
\hline 150 & 8 & 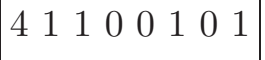 & 6 & $\begin{array}{llllllll}3 & 1 & 1 & 0 & 0 & 0 & 1 & 0\end{array}$ & 6 & $\begin{array}{llllllll}3 & 1 & 0 & 0 & 1 & 1 & 0 & 0\end{array}$ & 6 & $\begin{array}{lllllll}3 & 0 & 1 & 1 & 1 & 0 & 0\end{array}$ \\
\hline 151 & 8 & 41101010 & 4 & 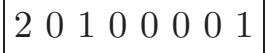 & 6 & $\begin{array}{llllllll}3 & 1 & 0 & 1 & 1 & 0 & 0 & 0\end{array}$ & 8 & 401111100 \\
\hline 152 & 10 & 5201010110 & 4 & 2100000001 & 4 & 20000111000 & 6 & $\begin{array}{lllllll}3 & 1 & 0 & 0 & 0 & 1 & 0\end{array}$ \\
\hline 153 & 7 & 13101100 & 5 & 12011000 & 6 & 13001010 & 8 & 141010001 \\
\hline 154 & 7 & 13011100 & 6 & 13100100 & 6 & 133001010 & 8 & 140011101 \\
\hline 155 & 8 & 14011010 & 3 & $\begin{array}{llllllll}0 & 1 & 0 & 1 & 0 & 1 & 0 & 0\end{array}$ & 4 & $\begin{array}{llllllll}0 & 2 & 0 & 1 & 0 & 0 & 0 & 1\end{array}$ & 6 & 131010000 \\
\hline 156 & 8 & 4101010110 & 4 & 20101001000 & 4 & 20001110000 & 6 & $\begin{array}{llllllll}3 & 0 & 0 & 1 & 0 & 0 & 1 & 1\end{array}$ \\
\hline 157 & 8 & 40110110 & 4 & 2100000010 & 6 & $\begin{array}{llllllll}3 & 0 & 1 & 0 & 0 & 0 & 1 & 1\end{array}$ & 6 & $\begin{array}{lllllllll}3 & 0 & 0 & 1 & 0 & 1 & 0\end{array}$ \\
\hline 158 & 10 & 52011100 & 4 & $\begin{array}{llllllll}2 & 1 & 0 & 0 & 0 & 0 & 1 & 0\end{array}$ & 6 & 31110001000 & 6 & $\begin{array}{llllllll}3 & 1 & 0 & 0 & 1 & 0 & 0 & 1\end{array}$ \\
\hline
\end{tabular}

Open Access. This article is distributed under the terms of the Creative Commons Attribution License which permits any use, distribution and reproduction in any medium, provided the original author(s) and source are credited.

\section{References}

[1] P. Candelas, X. De La Ossa, A. Font, S.H. Katz and D.R. Morrison, Mirror symmetry for two parameter models. I., Nucl. Phys. B 416 (1994) 481 [hep-th/9308083] [InSPIRE].

[2] A. Klemm, W. Lerche and P. Mayr, K3 fibrations and heterotic type-II string duality, Phys. Lett. B 357 (1995) 313 [hep-th/9506112] [INSPIRE].

[3] S. Kachru and C. Vafa, Exact results for $N=2$ compactifications of heterotic strings, Nucl. Phys. B 450 (1995) 69 [hep-th/9505105] [INSPIRE].

[4] A. Sen and C. Vafa, Dual pairs of type-II string compactification, Nucl. Phys. B 455 (1995) 165 [hep-th/9508064] [INSPIRE].

[5] P.S. Aspinwall and J. Louis, On the ubiquity of K3 fibrations in string duality, Phys. Lett. B 369 (1996) 233 [hep-th/9510234] [INSPIRE].

[6] G. Aldazabal, A. Font, L.E. Ibáñez and F. Quevedo, Chains of $N=2, D=4$ heterotic type-II duals, Nucl. Phys. B 461 (1996) 85 [hep-th/9510093] [INSPIRE].

[7] B. Hunt and R. Schimmrigk, Heterotic gauge structure of type-II K3 fibrations, Phys. Lett. B 381 (1996) 427 [hep-th/9512138] [INSPIRE].

[8] P.S. Aspinwall and M. Gross, Heterotic-heterotic string duality and multiple K3 fibrations, Phys. Lett. B 382 (1996) 81 [hep-th/9602118] [INSPIRE]. 
[9] C. Gomez, R. Hernandez and E. Lopez, K3 fibrations and softly broken $N=4$ supersymmetric gauge theories, Nucl. Phys. B 501 (1997) 109 [hep-th/9608104] [INSPIRE].

[10] S. Hosono, B. Lian and S.-T. Yau, Calabi-Yau varieties and pencils of K3 surfaces, alg-geom/9603020 [INSPIRE].

[11] A. Avram, M. Kreuzer, M. Mandelberg and H. Skarke, Searching for K3 fibrations, Nucl. Phys. B 494 (1997) 567 [hep-th/9610154] [INSPIRE].

[12] S.B. Giddings, S. Kachru and J. Polchinski, Hierarchies from fluxes in string compactifications, Phys. Rev. D 66 (2002) 106006 [hep-th/0105097] [INSPIRE].

[13] V. Balasubramanian, P. Berglund, J.P. Conlon and F. Quevedo, Systematics of moduli stabilisation in Calabi-Yau flux compactifications, JHEP 03 (2005) 007 [hep-th/0502058] [INSPIRE].

[14] M. Cicoli, J.P. Conlon and F. Quevedo, General analysis of LARGE Volume Scenarios with string loop moduli stabilisation, JHEP 10 (2008) 105 [arXiv: 0805.1029] [INSPIRE].

[15] J.P. Conlon, F. Quevedo and K. Suruliz, Large-volume flux compactifications: moduli spectrum and D3/D7 soft supersymmetry breaking, JHEP 08 (2005) 007 [hep-th/0505076] [INSPIRE].

[16] F. Denef, M.R. Douglas and B. Florea, Building a better racetrack, JHEP 06 (2004) 034 [hep-th/0404257] [INSPIRE].

[17] R. Blumenhagen, S. Moster and E. Plauschinn, Moduli stabilisation versus chirality for MSSM like type IIB orientifolds, JHEP 01 (2008) 058 [arXiv:0711.3389] [INSPIRE].

[18] A. Collinucci, M. Kreuzer, C. Mayrhofer and N.-O. Walliser, Four-modulus 'Swiss Cheese' chiral models, JHEP 07 (2009) 074 [arXiv:0811.4599] [INSPIRE].

[19] M. Berg, M. Haack and E. Pajer, Jumping through loops: on soft terms from large volume compactifications, JHEP 09 (2007) 031 [arXiv:0704.0737] [INSPIRE].

[20] M. Cicoli, J.P. Conlon and F. Quevedo, Systematics of string loop corrections in type IIB Calabi-Yau flux compactifications, JHEP 01 (2008) 052 [arXiv: 0708.1873] [INSPIRE].

[21] M. Cicoli, String loop moduli stabilisation and cosmology in IIB flux compactifications, Fortsch. Phys. 58 (2010) 115 [arXiv:0907.0665] [INSPIRE].

[22] M. Cicoli and F. Quevedo, String moduli inflation: an overview, Class. Quant. Grav. 28 (2011) 204001 [arXiv:1108.2659] [INSPIRE].

[23] M. Cicoli, C. Burgess and F. Quevedo, Fibre inflation: observable gravity waves from IIB string compactifications, JCAP 03 (2009) 013 [arXiv:0808.0691] [INSPIRE].

[24] C. Burgess et al., Non-standard primordial fluctuations and nonGaussianity in string inflation, JHEP 08 (2010) 045 [arXiv: 1005.4840] [INSPIRE].

[25] M. Cicoli and A. Mazumdar, Reheating for closed string inflation, JCAP 09 (2010) 025 [arXiv: 1005.5076] [INSPIRE].

[26] M. Cicoli and A. Mazumdar, Inflation in string theory: a graceful exit to the real world, Phys. Rev. D 83 (2011) 063527 [arXiv:1010.0941] [INSPIRE].

[27] L. Anguelova, V. Calo and M. Cicoli, LARGE volume string compactifications at finite temperature, JCAP 10 (2009) 025 [arXiv:0904.0051] [INSPIRE].

[28] M. Cicoli, M. Goodsell, J. Jaeckel and A. Ringwald, Testing string vacua in the lab: from a hidden CMB to dark forces in flux compactifications, JHEP 07 (2011) 114 [arXiv: 1103.3705] [INSPIRE]. 
[29] M. Cicoli, C. Burgess and F. Quevedo, Anisotropic modulus stabilisation: strings at LHC scales with micron-sized extra dimensions, JHEP 10 (2011) 119 [arXiv:1105.2107] [INSPIRE].

[30] N. Arkani-Hamed, S. Dimopoulos and G. Dvali, The hierarchy problem and new dimensions at a millimeter, Phys. Lett. B 429 (1998) 263 [hep-ph/9803315] [INSPIRE].

[31] I. Antoniadis, N. Arkani-Hamed, S. Dimopoulos and G. Dvali, New dimensions at a millimeter to a Fermi and superstrings at a TeV, Phys. Lett. B 436 (1998) 257 [hep-ph/9804398] [INSPIRE].

[32] Y. Aghababaie, C. Burgess, S. Parameswaran and F. Quevedo, Towards a naturally small cosmological constant from branes in $6-D$ supergravity, Nucl. Phys. B 680 (2004) 389 [hep-th/0304256] [INSPIRE].

[33] Y. Aghababaie et al., Warped brane worlds in six-dimensional supergravity, JHEP 09 (2003) 037 [hep-th/0308064] [INSPIRE].

[34] R. Blumenhagen and M. Schmidt-Sommerfeld, Power towers of string instantons for $N=1$ vacua, JHEP 07 (2008) 027 [arXiv:0803.1562] [INSPIRE].

[35] J.P. Conlon, A. Maharana and F. Quevedo, Towards realistic string vacua, JHEP 05 (2009) 109 [arXiv:0810.5660] [INSPIRE].

[36] R. Blumenhagen, J. Conlon, S. Krippendorf, S. Moster and F. Quevedo, SUSY breaking in local string/F-theory models, JHEP 09 (2009) 007 [arXiv:0906.3297] [INSPIRE].

[37] R. Blumenhagen, V. Braun, T.W. Grimm and T. Weigand, GUTs in type IIB orientifold compactifications, Nucl. Phys. B 815 (2009) 1 [arXiv:0811.2936] [InSPIRE].

[38] C. Beasley, J.J. Heckman and C. Vafa, GUTs and exceptional branes in F-theory - I, JHEP 01 (2009) 058 [arXiv:0802.3391] [INSPIRE].

[39] C. Beasley, J.J. Heckman and C. Vafa, GUTs and exceptional branes in F-theory - II. Experimental predictions, JHEP 01 (2009) 059 [arXiv:0806.0102] [INSPIRE].

[40] M. Kreuzer and H. Skarke, Complete classification of reflexive polyhedra in four-dimensions, Adv. Theor. Math. Phys. 4 (2002) 1209 [hep-th/0002240] [InSPIRE].

[41] Calabi Yau data: Tools and data for (toric) Calabi-Yau varieties, Landau-Ginzburg models, and related objects, http://hep.itp.tuwien.ac.at/ kreuzer/CY/.

[42] C. Mayrhofer, Compactifications of type IIB string theory and F-theory models by means of toric geometry, Ph.D. Thesis, Vienna University of Technology, Vienna Austria (2010), http://aleph.ub.tuwien.ac.at.

[43] M.R. Douglas and S. Kachru, Flux compactification, Rev. Mod. Phys. 79 (2007) 733 [hep-th/0610102] [INSPIRE].

[44] F. Denef, M.R. Douglas and S. Kachru, Physics of string flux compactifications, Ann. Rev. Nucl. Part. Sci. 57 (2007) 119 [hep-th/0701050] [INSPIRE].

[45] K. Oguiso, On algebraic fiber space structures on a Calabi-Yau 3-fold, Int. J. Math. 4 (1993) 439.

[46] H. Jockers and J. Louis, The effective action of D7-branes in $N=1$ Calabi-Yau orientifolds, Nucl. Phys. B 705 (2005) 167 [hep-th/0409098] [INSPIRE].

[47] H. Jockers and J. Louis, D-terms and F-terms from D\%-brane fluxes, Nucl. Phys. B 718 (2005) 203 [hep-th/0502059] [INSPIRE]. 
[48] M. Haack, D. Krefl, D. Lüst, A. Van Proeyen and M. Zagermann, Gaugino condensates and D-terms from D7-branes, JHEP 01 (2007) 078 [hep-th/0609211] [INSPIRE].

[49] M. Dine, N. Seiberg and E. Witten, Fayet-Iliopoulos terms in string theory, Nucl. Phys. B 289 (1987) 589 [inSPIRE].

[50] T.W. Grimm, M. Kerstan, E. Palti and T. Weigand, On fluxed instantons and moduli stabilisation in IIB orientifolds and F-theory, Phys. Rev. D 84 (2011) 066001 [arXiv:1105.3193] [INSPIRE].

[51] M. Cvetič, I. Garcia-Etxebarria and J. Halverson, Global F-theory models: instantons and gauge dynamics, JHEP 01 (2011) 073 [arXiv: 1003.5337] [INSPIRE].

[52] W. Fulton, Annals of mathematical studies. Vol. 131: Introduction to toric varieties, Princeton University Press, Princeton U.S.A. (1993).

[53] T. Oda, Ergebnisse der Mathematik und ihrer Grenzgebiete. Vol. 15: Convex bodies and algebraic geometry, Springer-Verlag, Berlin Germany (1987).

[54] H. Skarke, String dualities and toric geometry: an introduction, Chaos Solitons Fractals (1998) [hep-th/9806059] [INSPIRE].

[55] V. Bouchard, Lectures on complex geometry, Calabi-Yau manifolds and toric geometry, hep-th/0702063 [INSPIRE].

[56] M. Kreuzer, Toric geometry and Calabi-Yau compactifications, Ukr. J. Phys. 55 (2010) 613 [hep-th/0612307] [INSPIRE].

[57] K. Hori et al., Clay mathematics monographs. Vol. 1: Mirror symmetry, American Mathematical Society, Providence U.S.A. (2003), pg. 929.

[58] A.P. Braun, F-Theory and the Landscape of Intersecting D7-branes, arXiv:1003.4867 [INSPIRE].

[59] L.B. Anderson, Y.-H. He and A. Lukas, Monad bundles in heterotic string compactifications, JHEP 07 (2008) 104 [arXiv:0805.2875] [INSPIRE].

[60] M. Kreuzer and H. Skarke, PALP: a package for analyzing lattice polytopes with applications to toric geometry, Comput. Phys. Commun. 157 (2004) 87 [math/0204356] [INSPIRE].

[61] A.P. Braun and N.-O. Walliser, A new offspring of PALP, arXiv:1106.4529 [INSPIRE].

[62] G.-M. Greuel, G. Pfister, and H. Schönemann, Singular 3.0.2. A computer algebra system for polynomial computations. Centre for computer algebra, University of Kaiserslautern, Kaiserslautern Germany (2006), http://www.singular.uni-kl.de.

[63] F. Denef, Les Houches Lectures on constructing string vacua, arXiv:0803.1194 [INSPIRE].

[64] H. Grauert, Über Modifikationen und exzeptionelle analytische Mengen (in German), Math. Ann. 146 (1962) 331.

[65] C. Cordova, Decoupling Gravity in F-theory, arXiv:0910.2955 [INSPIRE].

[66] A. Collinucci, F. Denef and M. Esole, D-brane deconstructions in IIB orientifolds, JHEP 02 (2009) 005 [arXiv:0805.1573] [INSPIRE].

[67] V.V. Batyrev and L.A. Borisov, Mirror duality and string theoretic Hodge numbers, alg-geom/9509009 [INSPIRE].

[68] A. Collinucci, New F-theory lifts, JHEP 08 (2009) 076 [arXiv:0812.0175] [INSPIRE].

[69] M. Cicoli, C. Mayrhofer and R. Valandro, Moduli stabilisation for chiral global models, arXiv: 1110.3333 [INSPIRE]. 